\title{
Prediction of pan-solid tumor pembrolizumab benefit by integrating tumor mutation and gene expression profiling
}

Nickolay Khazanov

Strata Oncology

Melissa Shreve

Strata Oncology https://orcid.org/0000-0002-5983-4216

Laura Lamb

Strata Oncology

Daniel Hovelson

Strata Oncology

Marc Matrana

Ochsner Cancer Institute

Mark Burkard

University of Wisconsin-Madison

Eddy Yang

University of Alabama at Birmingham

William Edenfield

Prisma Health Greenville Memorial Hospital

Claire Dees

Lineberger Comprehensive Cancer Center

Adedayo Onitilo

ancer Care and Research Center, Marshfield Clinic Research Institute

Michael Thompson

Aurora Cancer Care, Advocate Aurora Health https://orcid.org/0000-0001-7930-4610

Gary Buchschacher

Kaiser Permanente Southern California

Alan Miller

SCL Health-CO

Alexander Menter

Kaiser Permanente Colorado

Benjamin Parsons

Gundersen Health System

Timothy Wassenaar 
Waukesha Memorial Hospital

Leon Hwang

Kaiser Permanente of the Mid-Atlantic States

J. Suga

Kaiser Permanente - Northern California

Robert Siegel

Bon Secours St. Franci

William Irvin, Jr.

Bon Secours St. Franci

Suresh Nair

Lehigh Valley Health Network

Jennifer Slim

MultiCare Regional Cancer Center

Kat Kwiatkowski

Strata Oncology

Khalis Mitchell

Strata Oncology

Tina Hu-Seliger

Strata Oncology

Stephanie Drewery

Strata Oncology

Andrew Fischer

Strata Oncology

Jennifer Hipp

Strata Oncology

Travis Reeder

Strata Oncology

Hana Vakil

Strata Oncology

Bryan Johnson

Strata Oncology

Daniel Rhodes

Strata Oncology

Scott Tomlins ( $\square$ scott.tomlins@strataoncology.com )

Strata Oncology https://orcid.org/0000-0001-8661-9821 
Keywords: immune response, oncology, pembrolizumab, biomarkers, pan tumor, tumor mutation burden, gene expression signature

Posted Date: January 5th, 2022

DOI: https://doi.org/10.21203/rs.3.rs-1225960/v1

License: (c) (1) This work is licensed under a Creative Commons Attribution 4.0 International License. Read Full License 


\title{
Prediction of pan-solid tumor pembrolizumab benefit by integrating tumor mutation burden and gene expression profiling
}

Nickolay Khazanov ${ }^{1}$, Melissa J. Shreve ${ }^{1}$, Laura E. Lamb ${ }^{1}$, Daniel H. Hovelson ${ }^{1}$, Marc R. Matrana ${ }^{2}$, Mark E. Burkard ${ }^{3}$, Eddy Shih-Hsin Yang ${ }^{4}$, William Jeffery Edenfield ${ }^{5}$, E. Claire Dees ${ }^{6}$, Adedayo A. Onitilo ${ }^{7}$, Michael Thompson ${ }^{8}$, Gary L. Buchschacher ${ }^{9}$, Alan M. Miller ${ }^{10}$, Alexander Menter ${ }^{11}$, Benjamin Parsons ${ }^{12}$, Timothy Wassenaar ${ }^{13}$, Leon C. Hwang ${ }^{14}$, J. Marie Suga ${ }^{15}$, Robert Siegel ${ }^{16}$, William Irvin, Jr. ${ }^{16}$, Suresh Nair ${ }^{17}$, Jennifer N. Slim ${ }^{18}$, Kat Kwiatkowski ${ }^{1}$, Khalis Mitchell ${ }^{1}$, Tina Hu-Seliger ${ }^{1}$, Stephanie Drewery ${ }^{1}$, Andrew Fischer ${ }^{1}$, Jennifer Hipp ${ }^{1}$, Travis Reeder ${ }^{1}$, Hana Vakil ${ }^{1}$, D. Bryan Johnson ${ }^{1}$, Daniel R. Rhodes ${ }^{1 *}$, Scott A. Tomlins ${ }^{1 *}$

${ }^{1}$ Strata Oncology, Ann Arbor, MI; ${ }^{2}$ Ochsner Cancer Institute, New Orleans, LA; ${ }^{3}$ University of Wisconsin Carbone Cancer Center, Madison, WI; ${ }^{4}$ O'Neal Comprehensive Cancer Center, University of Alabama at Birmingham School of Medicine, Birmingham, AL; ${ }^{5}$ Prisma Health Greenville Memorial Hospital, Greenville, SC; ${ }^{6}$ UNC Lineberger Comprehensive Cancer Center, Chapel Hill NC; ${ }^{7}$ Cancer Care and Research Center, Marshfield Clinic Research Institute, Marshfield, WI; ${ }^{8}$ Aurora Cancer Care, Advocate Aurora Health, Milwaukee WI; ${ }^{9}$ Kaiser Permanente Southern California, Los Angeles, CA; ${ }^{10}$ SCL Health-CO, Broomfield, CO; ${ }^{11}$ Kaiser Permanente Colorado, Lone Tree, CO; ${ }^{12}$ Gundersen Health System, La Crosse, WI; ${ }^{13}$ UW Health Cancer Center at ProHealth Care, Waukesha, WI; ${ }^{14}$ Kaiser Permanente of the Mid-Atlantic States, Rockville, MD; ${ }^{15}$ Kaiser Permanente - Northern California, Oakland, CA; ${ }^{16}$ Bon Secours St. Francis, Midlothian, VA; ${ }^{17}$ Lehigh Valley Health Network, Allentown, PA; ${ }^{18}$ MultiCare Regional Cancer Center - Tacoma, WA

\author{
Corresponding Author: \\ *Daniel R. Rhodes Ph.D. \\ Strata Oncology \\ 8192 Jackson Road, Suite A \\ Ann Arbor, MI 48103 USA \\ Phone: 734-527-0992 \\ email: daniel.rhodes@strataoncology.com \\ *Scott A. Tomlins, M.D., Ph.D. \\ Strata Oncology \\ 8192 Jackson Road, Suite A \\ Ann Arbor, MI 48103 USA \\ Phone: 734-527-0992 \\ email: scott.tomlins@strataoncology.com
}

\section{Manuscript word count: 6,816}

Running Title: Immune Response Score predicts pembrolizumab benefit

Keywords: immune response, oncology, pembrolizumab, biomarkers, pan tumor, tumor mutation burden, gene expression signature 


\begin{abstract}
Pembrolizumab is approved in many advanced solid tumor types, however predictive biomarkers and the proportion of pembrolizumab-benefiting patients vary. Biomarkers beyond PD-L1

immunohistochemistry, microsatellite instability (MSI) status, and tumor mutation burden (TMB) may improve benefit prediction. Here, leveraging treatment data (time to next treatment [TTNT]) and comprehensive genomic and quantitative transcriptomic profiling on routine tumor tissue from 708 patients (24 tumor types) collected in an ongoing observational trial (NCT03061305), we report a multivariate, integrative predictor of pan-solid tumor pembrolizumab benefit. The Immune Response Score (IRS) model, which includes TMB and quantitative $P D-1, P D-L 2, A D A M 12$ and $C D 4$ RNA expression, was confirmed as predictive through comparison of pembrolizumab TTNT with previous chemotherapy TTNT in a subset of 166 patients treated with both. Applying IRS to the entire NCT03061305 cohort ( $n=25,770$ patients), $13.2-30.7 \%$ of patients $(2.2-9.6 \%$ of patients outside of pembrolizumab approved tumor types [including TMB-High and MSI-High]) are predicted to benefit substantially from pembrolizumab. Hence, if prospectively validated, the IRS model may improve pembrolizumab benefit prediction across approved tumor types including patients outside of currently approved indications.
\end{abstract}


2 Checkpoint inhibitors (CPIs) have transformed cancer care with anti-PD-1 and anti-PD-L1 monoclonal

3 antibodies approved for use in multiple tumor types and pan tumor indications (microsatellite instability

4 high/mismatch repair deficient [MSI-H/dMMR] and tumor mutation burden $[\mathrm{TMB}] \geq 10$

5 mutations/megabase $[\mathrm{Muts} / \mathrm{Mb}])^{1-3}$. Improved biomarkers capable of predicting CPI benefit have the

6 potential to expand CPIs to additional patient populations outside of currently approved indications, and

7 to focus their application more effectively on likely responsive patients when alternative therapies exist.

8 PD-L1 immunohistochemistry (IHC) is required for treatment in many tumor types and serves as a

9 companion diagnostic biomarker; although, antibodies, staining platforms, PD-L1 expressing cells

10 included in scoring algorithms, and cutoffs vary across tumor types ${ }^{4-14}$. In addition, high TMB predicts

11 CPI response across multiple tumor types, although TMB determination approaches vary across studies

12 and tests, and only a fraction of TMB high (TMB-H) patients benefit ${ }^{15-24}$. For example, in the

13 KEYNOTE-158 study of 9 tumor types leading to pan-solid tumor approval of second-line

14 pembrolizumab in patients with $\mathrm{TMB} \geq 10 \mathrm{Muts} / \mathrm{Mb}$ by the FoundationOne companion diagnostic $(\mathrm{CDx})$

15 device, objective responses were observed in $37 \%, 13 \%$, and $6 \%$ of patients with $\mathrm{TMB} \geq 13 \mathrm{Muts} / \mathrm{Mb}$,

$16 \geq 10$ and $<13$ Muts/Mb, and $<10$ Muts/Mb, respectively ${ }^{25,26}$.

17 Numerous translational studies have demonstrated that PD-L1 expression, TMB (with clonal TMB

18 showing increased predictive ability vs. TMB methods including all somatic mutations), and other

19 immune related gene expression markers focusing on the tumor microenvironment (TME) are

20 independent predictors of response ${ }^{15,27-39}$; however, a single, integrative, clinically applicable and

21 validated test for treatment selection across solid tumors is lacking. Herein, leveraging pembrolizumab

22 real-world data (RWD) for treatment and comprehensive genomic and quantitative transcriptomic

23 profiling (CGqTP) data from the Strata Trial (NCT03061305) - an observational clinical trial evaluating

24 the impact of molecular profiling on patients with advanced solid tumors - we report the development of

25 an integrated clinical Immune Response Score (IRS) that predicts pembrolizumab response across solid 
26 tumors from small, real-world, formalin-fixed paraffin-embedded (FFPE) tumor tissue specimens.

27 Although prospective clinical validation is required, these results demonstrate that integrated CGqTP may 28 be able to increase the clinical benefit of the CPI pembrolizumab in patients with advanced solid tumors. 


\section{Cohort}

32 The Strata Clinical Molecular Database (SCMD) contains deidentified subject, molecular profiling,

33 treatment, and survival data captured from the Strata Trial (NCT03061305), a 500,000-patient

34 observational study for patients with advanced solid tumors. The Strata Trial has been reviewed and

35 approved by Advarra Institutional Review Board (IRB; IRB Pro00019183) prior to study start. At

36 enrolling health care systems, all adult patients with unresectable or metastatic solid tumors and available

37 FFPE tumor tissue were eligible. Although the protocol allowed enrollment of patients with rare early-

38 stage tumors, some analyses herein were restricted to patients with advanced (clinical stage III or IV)

39 disease as indicated at the time of enrollment, or unstaged tumor types. Prior antineoplastic therapy,

40 including start and stop dates, were collected for trial participants at the time of study entry.

41 Antineoplastic therapy data and survival status were prospectively collected for 3 years from the time of

42 enrollment and/or informed consent. Post-hoc power analysis was not performed to determine the sample

43 size. A case series analysis was performed herein focusing on the development of an integrative CGqTP

44 based pembrolizumab predictor, an exploratory aim of the trial. Patients in the SCMD tested by a version

45 of StrataNGS assessing TMB (see Biomarker Data below) with parallel gene expression testing data

46 from 25 January 2017 to 04 May 2021 were potentially eligible for analysis using a data cutoff of 19 May

$47 \quad 2021$.

48 To generate an integrative CGqTP based pembrolizumab predictor, patients in the SCMD with valid

49 StrataNGS derived TMB and gene expression data (including meeting the minimum $20 \%$ tumor content

50 requirement) and had greater than 1 month on pembrolizumab were identified as eligible. Real-world time

51 to next treatment (TTNT) was defined as the time in months from the initiation of a therapy to the date of

52 commencement of the next line of therapy (or date of death). Patients without an event (i.e., new therapy

53 start or death) were censored at their last date of medical history record update. Patients treated with

54 either pembrolizumab monotherapy or combination pembrolizumab plus chemotherapy were included. 
Samples collected after the start date of pembrolizumab were excluded. Source data verification in the

Strata Trial was performed for high-risk data fields such as demographics and treatment history per an approved Trial Monitoring Plan. Data completeness, consistency, and quality assurance checks were performed across the Strata electronic data capture (EDC) system per an approved Data Management Plan. Additional details on the Strata Trial experience and Strata molecular profiling have been

60 described ${ }^{40-42}$.

\section{Biomarker Data}

62 Multiplex PCR-based comprehensive genomic profiling (PCR-CGP), including TMB assessment, was 63 performed on FFPE solid tumor tissue using StrataNGS (Strata Oncology, Ann Arbor, MI). The current 64 version of StrataNGS is a 437 gene laboratory-developed test (LDT) for FFPE tumor tissue samples 65 performed on co-isolated DNA and RNA, which has been validated on over 1,900 FFPE tumor samples, 66 and is covered for Medicare beneficiaries ${ }^{41}$. While earlier StrataNGS versions were also used during the 67 study period, all had similar performance for the TMB assessment (and MSI) used herein ${ }^{42}$. In parallel, 68 immune gene expression was determined by analytically and clinically validated multiplex PCR-based 69 quantitative transcriptomic profiling via an investigational test performed on the same co-isolated RNA as 70 described ${ }^{40}$; different versions of this quantitative transcriptomic profiling test have been run in parallel 71 with StrataNGS (assessing 26, 46 and currently 103 expression targets), however only quantification of 72 RNA from the 46 target version was used herein. One or more exon-spanning PCR amplicons were 73 selected for each target gene and multiple housekeeping genes were included, with 3 pan-cancer stable 74 housekeeping genes used for clinical testing. Multiplex RNAseq was performed using Ampliseq after reverse transcription followed by Ion Torrent-based next-generation sequencing. Expression target transcripts were measured in normalized reads per million, whereby raw expression target read counts

77 were normalized by a factor that results in the median housekeeping gene expression value matching the same gene's standard reads per million in a reference FFPE normal cell line sample (GM24149) run in 
parallel with all clinically tested samples. ${ }^{40}$ Formal analytical validation of the multiplex PCR-based quantitative transcriptomic panel and the integrated StrataIO model will be described separately.

\section{Data Analysis}

TTNT across groups and treatments were visualized using the Kaplan Meier method with the log-rank test used to test TTNT curve differences. Correlation between TTNT and overall survival (OS) was calculated using Spearman's $p$ among patients with both a documented death event and at least two lines of therapy. Throughout this study, TMB-H was defined as $\geq 10$ Muts/Mb by StrataNGS, given the previous validation of TMB by StrataNGS and high concordance with TMB estimates from FoundationOne tissue testing ${ }^{41}$. All analyses were performed in python.

\section{Strata Clinical Molecular Database (SCMD) Validity Analysis}

Analyses to assess the clinical validity of the SCMD included an analysis of TTNT in first line, stage III and IV non-small cell lung cancer (NSCLC) adenocarcinoma stratified by the presence and absence of

91 standard- of-care (SOC), actionable alterations in EGFR (excluding the recent SOC actionable exon 20

92 insertions), $A L K$, and ROS1, as well as general TTNT in non-pembrolizumab treated patients receiving at

93 least two lines of antineoplastic therapy stratified by therapy class. (e.g., chemotherapy + chemotherapy, 94 hormonal therapy + chemotherapy, small molecule inhibitors + chemotherapy). For analysis of NSCLC 95 adenocarcinoma SOC alterations, the presence of both the genomic alteration and treatment history with 96 one of the FDA-approved targeted therapy for the alteration was considered as SOC treatment.

Real-World Progression Free Survival (rwPFS) as Measured by Time To Next Treatment (TTNT) Real-world progression free survival was measured by time to next treatment (TTNT) for patients within the SCMD database. Patient treatment history was standardized as described below, to ensure TTNT

101 calculations were performed appropriately for each treatment type. Medications were classified into 102 antineoplastic or non-antineoplastic treatments, and chemotherapy medications were defined as a subset 
of the antineoplastic treatments. Non-antineoplastic treatments were excluded from TTNT calculations;

104 patient treatment records with invalid or non-informative dates were also excluded (e.g., no start date or

105 start date in the future). Consecutive single-dose treatments were combined into a course of treatment

106 with a single start and end date. Since chemotherapy medications are frequently administered together,

107 any chemotherapy treatment(s) with a temporal overlap of $80 \%$ or more were merged into a single

108 treatment record. Lines of therapy were defined when a different therapy was started at, or after, the end

109 of another therapy (with the former therapy not being administered at any time after the latter). For

110 example, multiple starts and stops of the same therapy with a different therapy in between did not

111 delineate different lines of treatment.

112 To determine TTNT, an effective end date was defined for each course of treatment as either a) date of

113 record if treatment is ongoing, b) date of death if patient died while on treatment, or c) the latest available

114 end date. Furthermore, a likely progression event for the end of treatment was identified if either a) the

115 patient died during treatment or b) the patient started another antineoplastic treatment at the end of the

116 current treatment. TTNT was calculated as the difference, in months, between the start date and effective

117 end date of the treatment. Only records with 1 month or more anti-neoplastic TTNT were retained.

118 For analysis of pembrolizumab monotherapy vs combination therapy, a pembrolizumab course of

119 treatment was classified as combination therapy if there was $10 \%$ or more temporal overlap between the

120 pembrolizumab treatment and chemotherapy treatment(s).

\section{Immune Response Score (IRS) Model Development}

122 The association of TMB and 21 candidate immune and proliferation gene expression biomarkers with

123 pembrolizumab TTNT was determined using standard Cox proportional hazards regression. TMB

124 measurements were $\log _{2}$-transformed and gene expression measurements were $\log _{2}$-transformed and

125 median-centered per laboratory workflow (two PCR cycling conditions were used with the 46 gene

126 expression test) prior to analysis. For gene expression biomarkers with two independent expression 
127 amplicons on the 46 gene RNA test version ( $P D-1$ and $P D-L 1)$, results were averaged prior to inclusion in 128 the analysis (e.g. $P D-1$ composite). For multivariate model building, backward stepwise regression was 129 used, first including all variables in the model, then selectively removing the least significant variables so

130 long as the overall model significance improved. This approach was performed several times on a sub-

131 sampling and showed that most significant models [as measured by Akaike information criterion (AIC)]

132 contained no more than half-dozen factors. We therefore performed a brute-force search of all

133 combinations of expression targets to find the most significant factors. To minimize the risk of

134 overfitting, a final 5-factor model was selected. Individual patient IRS were derived from the Cox model

135 as:

$136 \quad \mathrm{IRS}=4.03 * \exp (0.29 * \mathrm{TMB}+0.15 * \mathrm{PD}-1+0.14 * \mathrm{PD}-\mathrm{L} 2-0.14 * \mathrm{CD} 4-0.07 * \mathrm{ADAM} 12)$

137 where an IRS of 10 is equal to the median hazard rate observed in the dataset, values greater than 10

138 represent decreased hazard (i.e., more benefit from pembrolizumab) and values less than 10 represent

139 increased hazard (i.e., less benefit from pembrolizumab). We assigned patients to one of three IRS groups

140 to compare patient outcomes (i.e., Low $(\mathrm{L})<8.5,8.5 \leq$ Intermediate $(\mathrm{I})<11.9$, and High $(\mathrm{H}) \geq 11.9)$

141 through dividing the dataset into 8 equal IRS bins and combining bins based on overlapping TTNT

142 curves. Cox proportional hazards models were utilized to examine the interaction between

143 pembrolizumab vs. prior chemotherapy TTNT within the same patient and IRS as a continuous variable.

144 The likelihood ratio test for interaction compared the reduced model, which excluded the IRS by

145 treatment interaction, with the competing full model, which included the IRS by treatment interaction. 


\section{RESULTS}

\section{Clinical Molecular Data}

150 The Strata Trial (NCT03061305) is an observational clinical trial evaluating the impact of tumor

151 molecular profiling for patients with advanced solid tumors. De-identified demographic, clinical and

152 molecular data from patients in the Strata Trial is maintained in the Strata Clinical Molecular Database

153 (SCMD). With a data-cutoff of 19 May 2021, the SCMD contains clinical and molecular data from

15439,252 unique patients with stage III or IV solid tumors (from 28 tumor types) enrolled from 25 United

155 States health care systems who had routine FFPE tumor tissue molecularly profiled by the StrataNGS

156 CGP test ${ }^{41,42}$ with 7,978 patients (from 28 tumor types) having treatment data from at least one

157 antineoplastic agent.

158 For all SCMD patients, antineoplastic treatment start and stop dates (for all prior therapies and up to 3

159 years after Strata trial enrollment) were obtained from automated electronic health record queries or

160 manual entry; data was updated regularly by submitting institutions, and date of death was obtained

161 similarly. Real-world TTNT was determined directly from treatment start and stop dates for each line of

162 therapy. Among the 7,978 patients, the median follow-up from start of first treatment and Strata trial

163 enrollment was 11 months [interquartile range (IQR) 4-24 months] and 5 months (IQR 1-11 months),

164 respectively. The median number of total therapies and lines of therapy per patient was 2 (both IQR 1-3),

165 with a median of 1 total therapy and 1 line of therapy (both IQR 1-2) after Strata trial enrollment. As

166 expected, in patients who had at least two lines of therapy, median TTNT was shorter with each

167 subsequent line of therapy (Figure S1).

168 Given the substantial proportion of patients in the SCMD with NSCLC and extensive previous

169 characterization of molecular subtypes and associated therapies, we leveraged the NSCLC cohort to

170 assess the validity of using the SCMD to support this study. Of the 7,978 total patients, $1,173(14.7 \%)$ had

171 NSCLC, with a median age at enrollment of 65 years (IQR 60-73), 51.8\% were women, 56.4\% were 
172 white, and $8.0 \%$ had NSCLC squamous cell carcinoma, similar to data reported for patients in the initial

173 report from the Flatiron/Foundation Medicine clinical molecular database ${ }^{17}$. At enrollment, of the 1,173

174 NSCLC patients in the SCMD, 24.8\% and 75.2\% had stage III and IV cancer, respectively. Of note, in

175139 patients with NSCLC adenocarcinoma harboring standard of care (SOC) alterations in $E G F R, A L K$ or

176 ROS1, 84\% received at least one matched targeted therapy, while only 68 of $935(7.2 \%)$ patients without

177 SOC EGFR, ALK or ROS1 alterations received one or more of these targeted therapies. These treatment

178 results contrast with the initial report of the Flatiron/Foundation Medicine clinical molecular database,

179 where only 480 of 737 (65\%) patients with NCCN-driver alterations in EGFR and $A L K$ rearrangements

180 received targeted therapy after advanced NSCLC diagnosis, while $26 \%$ of EGFR inhibitor treatment was

181 in patients without an $E G F R$ alteration ${ }^{17}$. In part this may be due to the contemporary nature of our series,

182 as for example, 57 of $81(70 \%)$ patients with EGFR SOC alterations in the SCMD treated with EGFR

183 TKI received osimertinib (or an osimertinib containing combination regimen) as their first line of EGFR

184 TKI therapy, and 26 of 28 (93\%) patients with ALK SOC alterations treated with ALK TKIs received

185 alectinib or brigatinib as their first line of ALK TKI therapy. Additional details and NSCLC analyses

186 supporting the validity of TTNT and the SCMD are shown in Figure S2.

\section{Biomarkers of Pembrolizumab Benefit Analysis}

188 We have previously demonstrated that molecular alteration frequency in the first $\sim 30,000$ patients

189 enrolled in the Strata Trial ${ }^{42}$ was similar to that observed in the Memorial Sloan Kettering single

190 institution pan-cancer profiling effort, MSK-IMPACT ${ }^{43}$, supporting the generalizability of the SCMD.

191 Herein, to assess general associations and develop an integrative CGqTP tumor-agnostic tumor

192 pembrolizumab predictive biomarker, we first limited results to the 5,233 patients in the SCMD who met

193 the following criteria: TMB measurements from StrataNGS testing (including meeting the overall $20 \%$

194 tumor content requirement), immune gene expression quantification from an investigative multiplex PCR

195 based transcriptomic profiling test, and treatment for at least one month with at least one antineoplastic

196 agent. Of these 5,223 patients, 708 (13.5\%) were treated with pembrolizumab. As shown in Figure 1a, 
this cohort was comprised of patients with 24 tumor types, with NSCLC accounting for 293 (41.4\%).

198 Real-world TTNT was inferred for each patient as the time from starting pembrolizumab to the time of 199 stopping pembrolizumab and starting a new therapy or death. To establish the appropriateness of TTNT 200 for studying pembrolizumab treatment outcomes, TTNT was compared to overall survival (OS). As 201 shown in Figure S3a, the overall correlation (Spearman $\rho=0.61$ ) was impacted by two outliers, one of 202 which was a patient with metastatic melanoma who was briefly treated with pembrolizumab, then 203 ipilimumab + nivolumab, prior to an extended course with imatinib (the patient harbored two VUS in 204 KIT; FigureS3a blue box), while the other was a patient with metastatic NSCLC harboring an EML4$205 A L K$ fusion by StrataNGS testing who was briefly treated with pembrolizumab and chemotherapy before 206 prolonged treatment with crizotinib and lorlatinib (FigureS3a red box); excluding these two patients, 207 TTNT and OS were more strongly correlated (Spearman $\rho=0.75$ ). Lastly, to confirm the validity of $\geq 10$ 208 Muts/Mb from StrataNGS testing to define TMB-H, we demonstrated that TMB-H patients $(n=208)$ had 209 significantly longer pembrolizumab TTNT vs. TMB-L patients $(n=500$; median TTNT $>24$ months vs. $21010.3, \log$ rank $p<0.0001$; Figure S3b).

211 To identify potential biomarkers of pembrolizumab benefit, we first considered 21 candidate immune and 212 proliferation gene expression biomarkers assessed across clinical RNA tests run in parallel with the 213 StrataNGS CGP test (which generates TMB). Importantly, target gene expression (in normalized reads 214 per million [nRPM]), were highly correlated from independent amplicons targeting different exons of $P D$ $215 L 1(\mathrm{n}=25,769$ samples, concordance correlation coefficient $=0.83)$ and $P D-1(\mathrm{n}=25,769$ samples,

216 concordance correlation coefficient $=0.78$ ). Likewise, expression profiles of these 21 genes across 27

217 directly comparable tumor types were highly correlated between 8,424 TCGA tumors and 18,062 Strata

218 RNA component profiled tumors (median Spearman $\rho=0.871$ for all candidate genes; Table S1). We

219 therefore assessed the association of pembrolizumab TTNT with StrataNGS derived TMB and the 21 220 candidate immune gene expression biomarkers. As shown in Table 1, significant $(p<0.01)$ univariate 221 predictors included TMB $(\mathrm{HR}=0.77 ; p<0.0001)$, composite $P D-L 1$ expression $(\mathrm{HR}=0.90 ; p=0.0007)$, 
composite $P D-1$ expression $(\mathrm{HR}=0.90 ; p=0.0011), P D-L 2$ expression $(\mathrm{HR}=0.91 ; p=0.005)$ and

TNFRSF9 expression $(\mathrm{HR}=0.92 ; p=0.007)$.

224 To develop an integrative pembrolizumab benefit predictor, we performed a multi-part process. First, we

225 performed backwards stepwise regression to fit a multivariate Cox proportional hazards model, iterating

226100 times on randomly selected two-thirds of the dataset. TMB, $P D-1$ and $P D-L 2$ were the three most

227 frequently included variables across the 100 models. Hence, we then used a brute-force approach, locking

228 in these three variables and using backwards stepwise regression adding in 1, 2 or 3 of the remaining 229 candidate variables, iterating 100 times on randomly selected $2 / 3$ rds of the dataset, and evaluating the 230 Akaike's Information Criteria (AIC) and Bayesian Information Criterion (BIC) of the trained model on

231 the held-out $1 / 3$ of the dataset. As additional components increased the BIC with minimal decrease in 232 AIC, we chose a five variable multivariate model including TMB, PD-1, PD-L2, CD4, and ADAM12. We 233 therefore entered all five variables into model selection 100 times on randomly selected two-thirds of the 234 dataset and determined the model coefficients, confirming that that model coefficients developed in the 235 full 708 patient cohort were stable, and these coefficients were used in the final integrative model (Figure 236 S4a). Multivariate analysis on only the final five variable set confirmed that all five biomarkers were 237 independent predictors of pembrolizumab treatment outcome (Table 1). Notably, $P D-L 1$ was not included 238 in the final model and forced addition to the five variable model had essentially no impact on

239 performance, even when trained on the full 708 patient cohort (Figure S4b). As shown in Figure S4c, 240 TMB was minimally correlated with all final model gene expression biomarkers (Spearman $\rho=-0.106$ $241[C D 4]$ to $-0.0 .015[P D-1])$, while correlation of individual gene expression biomarkers ranged from $\rho=$ $2420.217(A D A M 12$ vs. $P D-1)$ to $\rho=0.675(P D-L 2$ vs. $C D 4)$.

\section{Integrative Immune Response Score (IRS) Development}

244 To evaluate the potential of the multivariate model to predict pembrolizumab treatment outcome in 245 patients, we derived individual Immunotherapy Response Scores (IRS) from the final five variable model, 246 assigned the 708 patients to one of three IRS groups based on potential clinical utility (see Methods; IRS- 
High [-H; n=266], IRS-Intermediate [-I; n=176] and IRS-Low [-L; n=266]; IRS-H associated with greatest benefit of pembrolizumab), and compared group outcomes. Kaplan Meier analysis of pembrolizumab TTNT showed that treatment outcome varied widely across groups, with median TTNT 250 ranging from $>24$ months in IRS-H, to 17.5 months in IRS-I, to 7 months in IRS-L (log-rank test 251 p $<0.0001$ IRS-H vs. -L; Figure 1b). To compare to OS, Kaplan Meier analysis was also performed with 252 respect to time-to-death, censoring patients with respect to latest date of follow-up, producing similarly 253 significant difference in outcome for IRS-H vs. -L ( $n=707, \log$-rank test $\mathrm{p}<0.0001$; Figure S5a).

To establish the IRS model as predictive and not prognostic, we evaluated a chemotherapy comparator cohort, consisting of the most recent previous chemotherapy line from the 166 of $708(23 \%)$ pembrolizumab patients with documented chemotherapy treatment prior to monotherapy pembrolizumab.

258 While chemotherapy median TTNT was similar across all three IRS groups at 7.0-8.2 months (Figure 259 1c), pembrolizumab had significantly longer TTNT than chemotherapy in IRS-H (median TTNT $>24$ 260 months vs. 7.1 months; log-rank $p$ value $<0.001$ ) and IRS-I (median TTNT 13.5 months vs. 8.2 months, $261 \log$-rank $p$ value 0.02 ), but no significant difference was observed for pembrolizumab vs. chemotherapy 262 TTNT in IRS-L (median TTNT 5.8 vs. 7.1 months; log-rank p value $=0.65$ ). The test for interaction 263 between pembrolizumab vs. previous chemotherapy treatment and continuous IRS was significant 264 (likelihood ratio test for interaction $\mathrm{p}<0.005$ ), confirming the predictive nature of the IRS biomarker. 265 Through a similar analysis using continuous TMB (instead of the IRS model), TMB alone was also 266 confirmed as a predictive biomarker of pembrolizumab TTNT (likelihood ratio test for interaction $267 \mathrm{p}<0.005)$. However, IRS (expression component + TMB) had significantly greater predictive ability than 268 TMB alone (likelihood ratio test between models, $\mathrm{p}=0.04$ ). As in the overall cohort, TTNT and OS 269 comparisons were similar between IRS-H vs. -L patients in this predictive analysis (in addition to a cohort 270 of 201 patients considering pembrolizumab monotherapy or combination therapy vs. previous 271 chemotherapy), although IRS-I did not appear to be a separate group in these comparisons (Figure S5b- 
d). Taken together, these results demonstrate the predictive nature of the IRS model for pembrolizumab benefit prediction vs. an internal chemotherapy comparator and highlight the benefit of combining CGP biomarkers (TMB) with parallel quantitative gene expression of the tumor and TME to improve performance.

\section{Robustness of IRS model to potential confounding factors}

277 We next evaluated potential factors that could confound the utility of IRS [i.e., tumor type, therapy type 278 (monotherapy vs combination therapy) and TMB status]. First, we compared pembrolizumab TTNT in the 293 patients with NSCLC (41.4\%) to 415 patients with other tumor types (48.6\%) across IRS groups and found no significant differences (median TTNT $>24$ vs. $>24$, log-rank $\mathrm{p}=0.13$ for IRS-H; median TTNT $>24$ vs. 13.5 , log-rank $\mathrm{p}=0.19$ for IRS-I; median TTNT 7.2 vs. 6.1 , log-rank $\mathrm{p}=0.10$ for IRS-L; Figure 2a). Then we compared pembrolizumab TTNT in the 481 patients treated with pembrolizumab monotherapy (67.9\%) to 227 patients treated with pembrolizumab plus chemotherapy (combination therapy) (32.1\%), and found no significant difference in any IRS risk group (median TTNT $>24$ vs. $>24$, $\log$-rank $\mathrm{p}=0.87$ for IRS-H; median TTNT $>17.5$ vs. 15.6 , log-rank $\mathrm{p}=0.77$ for IRS-I; median TTNT 6.4

286 vs. 7.2, $\log$-rank p=0.20 for IRS-L; Figure 2b). Lastly, given the pan-tumor approval of pembrolizumab in TMB-H patients, if the IRS risk groups were exactly overlapping with TMB status, the IRS would have no clinical utility. Therefore, we examined the predictive effect of IRS groups among the 208 (29.4\%) TMB-H patients and 500 (70.6\%) TMB-L patients. While $167(80 \%), 28(13 \%)$ and $13(6 \%)$ of the 208 TMB-H patients were IRS-H, -I, and -L, respectively, the TMB-H/IRS-H group still had significantly longer pembrolizumab TTNT compared to TMB-H/IRS-L (median TTNT >24 months vs. 10.4 months; $\log$ rank p-value $=0.001$, Figure 3c), demonstrating the added predictive value of immune gene expression, even among TMB-H patients. Among the 500 TMB-L patients, 99 (20\%), 148 (30\%) and 253

294 (50\%) were IRS-H, -I, and -L, respectively. IRS robustly stratified pembrolizumab TTNT, with median pembrolizumab TTNT of 20.8 months, and 7 months in IRS-H, and -L groups, respectively (log rank p- 
of pembrolizumab benefit. Together, these results demonstrate that the IRS biomarker is robust to tumor type, pembrolizumab monotherapy vs. combination chemotherapy treatment, and TMB status.

\section{Stability of IRS across temporal sample collection variability prior to CPI treatment}

301 Tissue based TMB has recently been shown to be stable for nearly all patients with advanced cancer

302 through whole genome sequencing of sequential tissue samples, ${ }^{44}$ however less is known about the

303 stability of an integrative CGqTP model predicting pembrolizumab benefit. Hence, we first confirmed

304 that in the 426 total patients treated with both chemotherapy (regardless of whether chemotherapy was

305 pre- or post- pembrolizumab treatment) and pembrolizumab, the timing of sample collection (pre-

306 chemotherapy and pembrolizumab vs. post-chemotherapy but prior to pembrolizumab) did not

307 significantly impact median pembrolizumab TTNT across IRS groups (Figure S6a). Next, we directly

308 assessed IRS stability across patients in the SCMD with sequentially tested tissue samples. As analyses

309 presented thus far were limited to the most recently tested sample per patient (if testing had been

310 performed more than once) and to patients who started pembrolizumab after the collection date of the

311 included sample, we therefore identified 69 total patients in the SCMD who 1) had valid IRS scores from

312 two specimens with different collection dates, 2) were confirmed to be of clonal origin as part of routine

313 StrataNGS clinical testing, and 3) did not have CPI therapy starting between the collection dates of the

314 samples. As shown in Figure S6b, the integrative IRS model scores were highly correlated (Pearson

$315 r=0.75$, respectively) in paired specimens, and only two $(3 \%)$ patients $(\mathrm{n}=2)$ moved from the IRS-H to $-\mathrm{L}$

316 (or vice versa), supporting the stability of the IRS across temporal sampling in the absence of checkpoint

317 inhibitor therapy. Lastly, we assessed the performance of IRS in 84 patients who otherwise would have

318 been included in the 708 total patient discovery cohort described above, but had their sample collected

319 after starting pembrolizumab. Hypothesizing that CGP testing in this clinical scenario would usually be

320 performed as the patient was progressing on pembrolizumab, we predicted that IRS would be minimally

321 predictive of pembrolizumab TTNT. In these 84 patients, continuous IRS was not predictive of 
pembrolizumab TTNT ( $\mathrm{p}=0.61$ ), with median pembrolizumab TTNT of 15.9 vs. 15.6 months in IRS-H vs. -L, log rank p=0.92, Figure S6c). Together, these results support the stability and validity of IRS in sequential tumor tissue samples collected prior to CPI treatment.

\section{Pan Solid Tumor Distribution of IRS groups}

326 Although future studies are required to prospectively validate IRS performance for routine clinical use, 327 we sought to leverage IRS distributions across tumor types (and pan-cancer biomarkers) in the entire 328 SCMD to understand the potential impact of IRS both within and outside of currently approved 329 pembrolizumab indications. Thus, we determined IRS for the 25,770 patients in the SCMD 330 (NCT03061305) with valid TMB and gene expression data, with 13.2\%, 17.5\%, and 69.3\% of all patients 331 classified as IRS-H, -I, and -L, respectively (Figure 3a). Pembrolizumab approved tumor types (without 332 consideration of PD-L1 IHC status) had a substantially higher proportion (22.1\% vs. 7.1\%) of IRS-H 333 patients than non-pembrolizumab approved tumor types, as well as a higher proportion (23.6\% vs. $13.4 \%)$ 334 of IRS-I group patients (Figure 3b). Tumor types with the highest proportion of IRS-H group patients 335 include several known to be highly responsive to CPIs, including melanoma, non-melanoma skin cancer, 336 NSCLC, lung small cell carcinoma (Lung - Other), and bladder (urothelial) tumors (Figure 3c). We next 337 examined the pan-solid tumor distribution of IRS groups by TMB status, given the pan-tumor approval of 338 pembrolizumab in TMB-H tumors. Whereas $91 \%$ of TMB-H patients were also IRS-H or -I, $22.0 \%$ of all 339 patients in the SCMD were IRS-H or -I and TMB-L (vs. 8.7\% IRS-H or -I and TMB-H), demonstrating 340 that while TMB-H identifies most IRS-I/-H patients, IRS identifies a larger set of TMB-L patients 341 predicted to benefit from pembrolizumab (Figure 3d). Finally, to estimate the overall proportion of 342 patients with solid tumors who might benefit from pembrolizumab outside of currently approved tumor 343 types and biomarkers, we stratified the SCMD population by all pembrolizumab approved indications 344 (pembrolizumab approved tumor types, TMB-H, or MSI-H as approved). As shown in Figure 3e, if 345 prospectively validated, an additional $2.2-9.6 \%$ of patients (2.2\% IRS-H and $7.4 \%$ IRS-I) with solid 
tumors outside of currently approved indications are predicted to have substantial benefit from

347 pembrolizumab.

\section{Discussion}

349 Leveraging a robust clinical molecular database from the StrataTrial (NCT03061305), we developed a

350 highly significant, integrative, multivariate Immune Response Score (IRS) model that combined TMB

351 and quantitative immune gene expression to predict real-world pembrolizumab treatment outcomes in 708

352 patients from 28 solid tumor types. IRS model inputs were generated from simultaneously performed,

353 clinically validated, multiplex PCR based DNA and RNA NGS (StrataNGS CGP and a separate RNA

354 panel for quantitative gene expression $)^{40-42}$. These assays were performed on co-isolated DNA and RNA

355 and share the same key sample input requirements defined from over 30,000 consecutively received FFPE

356 tumor samples for CGP testing: $\geq 20 \%$ tumor content and $2 \mathrm{~mm}^{2}$ tumor surface area (from $10 \times 5$ um FFPE

357 sections $)^{41,42}$. Of note, only $38.3 \%$ of samples in the development cohort included herein, and $38.8 \%$ of

358 the 25,770 total patients in the SCMD used to assess IRS distribution, met the minimum tumor surface

359 area requirements $\left(\geq 25 \mathrm{~mm}^{2}\right)$ of FoundationOne $\mathrm{CDx}^{45}$, the FDA approved companion diagnostic device

360 to identify TMB-H tumors.

361 The IRS model predicts an individual patient's likelihood of benefit with pembrolizumab therapy.

362 Patients were grouped into three categories (IRS-H, IRS-I, and IRS-L) based on pembrolizumab TTNT as

363 a measure for potential clinical utility, with IRS-H patients having median pembrolizumab TTNT $>24$

364 months, while IRS-L patients had median pembrolizumab TTNT of 7 months. Critically, in the subset of

365166 patients treated with pembrolizumab monotherapy who had prior chemotherapy treatment, we

366 confirmed the predictive nature of the IRS model, as IRS-H patients had significantly longer TTNT on

367 pembrolizumab vs. their immediately preceding chemotherapy treatment (median TTNT $>24$ months vs.

3687.1 months), whereas IRS-L patients did not (median TTNT 5.8 vs. 7.1), with a significant test for

369 interaction between continuous IRS and pembrolizumab vs. chemotherapy. Notably, the association of 
IRS with pembrolizumab TTNT was stable when stratified by NSCLC vs. other tumor types,

371 pembrolizumab monotherapy vs. combination therapy, TMB-H vs. TMB-L tumors, and pre- vs. post-

372 chemotherapy sample collection, suggesting that the model captures universal biological features of

373 pembrolizumab benefit. When applied to all 25,770 patients in the SCMD where IRS could be generated,

374 IRS-H was more frequent in tumor types known to derive benefit from CPI, but occurred in subsets of

375 nearly every tumor type. Outside of approved pembrolizumab tumor type indications, including TMB-H

376 and MSI-H pan cancer, 2.2\% patients in the SCMD were IRS-High, representing a conservative estimate

377 as many approved indications have PD-L1 IHC requirements. Hence, if subsequently validated in our

378 ongoing studies, the improved predictive clinical utility of the integrative IRS model would be

379 demonstrated to more accurately identify patients across tumor types with a high probability of response

380 to pembrolizumab over single biomarker approaches.

381 Intriguingly, an additional $7.4 \%$ of patients were IRS-I outside of tumor types with FDA-approved 382 pembrolizumab indications (also excluding MSI-H and TMB-H). Although these patients had 383 significantly longer monotherapy pembrolizumab TTNT vs. their previous chemotherapy (13.5 vs. 8.2 384 months) and the proportion of IRS-I patients in selected tumor types is consistent versus observed 385 response rates $(\mathrm{ORR})$ in early phase pembrolizumab (e.g.,13\% of SCMD patients with pancreatic cancer 386 are IRS-I versus an ORR of $10 \%$ in non-biomarker selected patients treated with pembrolizumab 387 monotherapy in the KEYNOTE-028 trial $^{36}$ ), tumor specific trials may be needed to demonstrate a clear 388 benefit of pembrolizumab in this subset of patients.

389 As the IRS was developed from a single integrative clinical platform using co-isolated DNA and RNA to 390 generate TMB and highly quantitative gene expression assessment of the tumor and TME from over 700 391 patients across 24 tumor types, the IRS model holds several potentially interesting biological insights.

392 First, TMB, $P D-1$ expression, and $P D-L 2$ expression were each independent predictors of pembrolizumab

393 benefit, indicating a multiplicative predictive effect across these biomarkers representing increased 394 antigenicity (TMB), the direct target of pembrolizumab ( $P D-1)$, and one of the two PD-1 interacting 
ligands $(P D-L 2)$. Notably, although $P D-L 1$ expression was predictive of pembrolizumab TTNT on univariate analysis, it was not an independent significant predictor identified through our multi-step

397 multivariate model developed process. In an exploratory analysis, when $P D-L 1$ RNA was added into the

398 IRS model and trained on the entire cohort, essentially no change in performance was observed,

399 consistent with its predictive ability being captured by the other model components. While PD-L1

400 evaluation by IHC is the current FDA-approved biomarker to predict pembrolizumab (or other CPI)

401 benefit either individually or in models ${ }^{45-47}$, expression varies by antibody clone and nearly all studies

402 show at least some responsive PD-L1-IHC low/negative patients, suggesting that other PD-1 ligands

403 beyond PD-L1, such as PD-L2, may be relevant for predicting clinical response ${ }^{30,48-51}$. Consistent with

404 this observation, in head and neck squamous cell carcinoma, PD-L2 expression by IHC predicted

405 pembrolizumab response and progression free survival independent of PD-L1 IHC status ${ }^{46}$. CD4 and

406 ADAM12 were both negative predictors of pembrolizumab TTNT in numerous model training iterations

407 and were significant in the final five variable IRS model. Although both effector CD8+ and CD4+ T cells

408 have been shown to express PD-1 ${ }^{47}, C D 8 A$ (which encodes CD8 and was included in our 21 candidate

409 genes), was more predictive of CPI benefit in a recent metanalysis of whole transcriptome data than either

$410 P D-L 1$ expression or the $\mathrm{T}$ cell inflamed gene expression signature ${ }^{15}$, and hence the inclusion of $C D 4$ as a

411 negative predictive factor in the final IRS model likely reflects at least in part the ratio of effector (CD8+)

412 to helper/regulatory CD4+ T cells in the TME.

413 Although less is known about the direct role of $A D A M 12$ in CPI response, it is highly expressed by cancer

414 associated fibroblasts CAFs — as shown through single cell sequencing studies and bulk tumor profiling-

415 as a driver of feed forward TGF- $\beta$ signaling, has been shown to act as a $\mathrm{T}$ cell co-stimulatory molecule

416 expressed on some regulatory T cells, and has been identified in a signature of negative response to ICI in

417 melanoma ${ }^{52-57}$. Of note, in colorectal cancer, where single cell sequencing demonstrated high ADAM12

418 expression in $\mathrm{CAFs}^{58}$, as well as urothelial carcinoma, TGF- $\beta$ signaling from CAFs has been shown to

419 drive T cell exclusion, a hallmark of low response to $\mathrm{ICI}^{59-63}$. Taken together, these results support 
additional investigation into a potential mechanistic role for $A D A M 12$ in ICI resistance, as well as

421 demonstrate the complementary nature of the integrative biomarkers in the IRS model, which integrates

422 measurement of tumor neo-antigenicity (TMB), with quantification of key tumor and TME biomarkers.

423 Current FDA-approved CPI biomarkers include PD-L1 IHC, TMB and MSI-H (although the latter

424 indication was initially approved without a companion diagnostic biomarker), however these biomarkers

425 have several practical challenges for clinical use including variations in assay parameters, platforms, and

426 predictive thresholds ${ }^{4,64-67}$. For example, although there are multiple tissue TMB assays commercially

427 available (LDTs, FDA cleared devices, and a single FDA approved device), TMB testing typically has a

428 large tissue requirement, which is frequently not feasible in patients with advanced cancers, and such

429 approaches do not allow for parallel assessment of gene expression biomarkers. Thus, there is a need for

430 optimized CPI biomarkers with improved predictive utility that can be developed into a scalable clinical

431 test that is applicable to nearly all cancer patients, including those with limited tumor tissue available. As

432 described herein, IRS addresses these needs by 1) co-isolating DNA and RNA to measure multiple classes

433 of biomarkers from the same tissue sample; 2) optimizing pembrolizumab treatment benefit prediction via

434 explainable model development using highly quantitative gene expression data in a large pan-tumor

435 cohort with real world treatment data; and 3) utilizing a clinically validated and scalable platform

436 developed for real world FFPE samples with minimal tumor size $\left(2 \mathrm{~mm}^{2} \text { tumor surface area }\right)^{41,42}$. In

437 comparison, only $38.8 \%$ of the 25,770 patients in the SCMD used to assess IRS distribution met the

438 minimum tumor surface area requirements $\left(\geq 25 \mathrm{~mm}^{2}\right)$ for FoundationOne CDx, the FDA approved

439 companion diagnostic device to identify TMB-H tumors ${ }^{45}$, suggesting that the majority of real-world

440 patients with advanced solid tumors have insufficient tumor samples to determine TMB.

441 Our analysis has several potential limitations. First, the real-world dataset was biased toward tumor types

442 for which pembrolizumab is FDA-approved or treatment was selected based on other biomarker results,

443 and thus, as expected, was enriched for patients benefiting from pembrolizumab. Indeed, the proportion of

444 patients in the IRS-High group was much higher in the pembrolizumab treatment cohort (38\%) than the 
broader tumor profiling dataset (13.2\%). Second, the TTNT endpoint likely includes some patients who

446 stopped treatment due to treatment toxicity or switching therapy to a more appropriate regimen based on

447 molecular results (as described above) and not disease progression, although this likely represents a

448 minority of events. Additionally, only advanced patients were eligible for the Strata Trial, but adjuvant

449 therapy cannot be directly excluded using our treatment data collection approach, with this limitation

450 being relevant for our pembrolizumab vs. prior chemotherapy analysis; however, this would tend to bias

451 against pembrolizumab TTNT being longer than adjuvant chemotherapy in patients with significant delay

452 between adjuvant chemotherapy and development of metastatic disease (and pembrolizumab treatment).

453 Additionally, although it is unclear if our model is applicable to other PD-1 monoclonal antibodies, PD-

454 L1 monoclonal antibodies, and/or combined PD-1/PD-L1 and CTLA4 antibody therapy, we focused on

455 pembrolizumab herein given the large amount of treatment data in our cohort across tumor types.

456 Likewise, future studies will also investigate whether inclusion of single gene-based DNA biomarkers

457 identified as potentially predictive in one or more tumor types (e.g. STK11, PBRM1, ARID1A, CDNK2A $A^{68-}$

45875 or additional immune related genes assessed on the current expanded quantitative expression panel run

459 in parallel with StrataNGS testing can improve the performance of the IRS model. Limited PD-L1 IHC

460 data was available for subjects in the SCMD, and hence we are not able to directly compare performance

461 of IRS and PD-L1 IHC for predicting pembrolizumab benefit; additionally, this limitation also biases

462 against the overall proportion of patients outside of currently approved indications predicted to benefit

463 from pembrolizumab by IRS, as herein we considered all patients in approved tumor types to be in an

464 approved indication, although in many tumor types only a minority of patients are approved for

465 pembrolizumab treatment based on PD-L1 IHC cutoffs. Notably, we chose to use standard multivariate

466 regression with a minimum number of variables versus other approaches that have included a larger

467 number of immune related genes ${ }^{27,34,39}$ or used more advanced machine learning approaches ${ }^{76}$ to leverage

468 the highly quantitative nature of CGqTP and minimize the risk of overfitting, as our model was trained

469 and characterized on the same dataset. Importantly, while we were able to use an internal chemotherapy

470 control cohort to determine the predictive nature of the biomarker, additional, pre-specified validation of 
the IRS model in an independent cohort will be required to establish the clinical utility of IRS groups for

472 predicting pembrolizumab benefit.

473 In summary, after demonstrating the face validity a clinical molecular database containing treatment data

474 and molecular profiling from a large observational trial of patients with advanced cancer, we report the

475 development of a biologically rational, integrative CGqTP based model of pembrolizumab benefit that is

476 robust to tumor type, TMB status, pembrolizumab monotherapy vs. combination therapy treatment, and

477 pre-pembrolizumab sample collection timing. Importantly, the IRS biomarker was developed from a

478 single clinically validated NGS platform capable of simultaneously performing comprehensive genomic

479 profiling (required for TMB but also for therapy options outside of CPI) and in parallel precise

480 quantification of tumor- and TME-relevant gene expression, providing a clear diagnostic pathway for

481 potential clinical application. IRS has potential application for both refining the use of pembrolizumab in

482 tumor types for which immunotherapy is indicated and therapeutic choice is present (as well as

483 monotherapy pembrolizumab vs. combination therapy as in NSCLC), as well as for guiding

484 pembrolizumab treatment decisions for patients outside of indicated tumor types. Most notably, IRS-High

485 patients treated with pembrolizumab had not reached median TTNT after 24 months (compared to a

486 median TTNT of 7.1 months on their prior line of chemotherapy), suggesting that this population may

487 benefit similarly to the TMB-H population identified in KEYNOTE-158 (29\% overall response rate; $66 \%$

488 of responders having a duration of response $\geq 24$ months). Herein, across the entire SCMD, $2.2 \%$ of

489 patients were IRS-H/TMB-L/not-MSI-H and outside of approved pembrolizumab approved tumor types,

490 and an additional 7.4\% were IRS-I. Hence, if further validated in additional cohorts, the IRS model has

491 the potential to markedly expand the benefit of pembrolizumab across solid tumors, addressing one of the

492 most important challenges in precision oncology. 
The authors thank Drs. Malek Safa (Kettering Cancer Center, Kettering, OH), Arvinda Padmanabhan (Baptist Health, Louisville, KY), Vallathucherry Harish (Hayworth Cancer Center at High Point Medical Center, High Point, NC), Abdul Hai Mansoor (Kaiser Permanente Northwest, Portland, Oregon), Dan Anderson (Metro-Minnesota Community Oncology Research Consortium, St. Louis Park, MN), William Schulz (UW-SwedishAmerican, Rockford, IL), Anneliese Gonzalez (UT Health - Memorial Hermann Cancer Institute, Houston, TX) as principal investigators and for their sites contribution to the study. We wish to dedicate this manuscript to our departed colleague, Dr. Michael Guarino (Christiana).

\section{AUTHOR CONTRIBUTIONS}

Conception and design: Kat Kwiatkowski, D. Bryan Johnson, Daniel R. Rhodes, Scott A. Tomlins,

Administrative support: Laura E. Lamb, Melissa J. Shreve

Provision of study materials or patients: Marc R. Matrana, Mark E. Burkard, Eddy Shih-Hsin Yang, William Jeffery Edenfield, E. Claire Dees, Adedayo A. Onitilo, Michael Thompson, Gary L. Buchschacher, Alan M. Miller, Alexander Menter, Benjamin Parsons, Timothy Wassenaar, Leon C. Hwang, J. Marie Suga, Robert Siegel, William Irvin, Jr., Suresh Nair, Jennifer N. Slim

Collection and assembly of data: Marc R. Matrana, Mark E. Burkard, Eddy Shih-Hsin Yang, William Jeffery Edenfield, E. Claire Dees, Adedayo A. Onitilo, Michael Thompson, Gary L. Buchschacher, Alan M. Miller, Alexander Menter, Benjamin Parsons, Timothy Wassenaar, Leon C. Hwang, J. Marie Suga, Robert Siegel, William Irvin, Jr., Suresh Nair, Jennifer N. Slim, Kat Kwiatkowski, Khalis Mitchell, Stephanie Drewery, Andrew Fischer, Jennifer Hipp, Travis Reeder, Hana Vakil

Data analysis and interpretation: Nickolay Khazanov, Daniel H. Hovelson, Tina Hu-Seliger, D. Bryan Johnson, Daniel R. Rhodes, Scott A. Tomlins

Manuscript writing: All authors

Final approval of manuscript: All authors

Accountable for all aspects of the work: All authors

\section{DATA AVAILABILITY}

Relevant data supportive of the figures, tables, and results of the paper are found in the Supplemental Materials. In the interest of protecting patient privacy and in accordance with applicable data sharing agreements and/or patient informed consent forms, the authors are restricted from making raw patient-level data publicly available. However, interested parties may contact the authors at BD@strataoncology.com to request access for research purposes, and such requests will be handled on a case-by-case basis. 
The IRS model algorithm is provided in the Methods.

COMPETING INTERESTS

532 Khazanov, Shreve, Lamb, Hovelson, Kwiatkowski, Mitchell, Hu-Seliger, Stephanie Drewery, Fischer, 533 Hipp, Reeder, Vakil, Johnson, Rhodes and Tomlins are/were equity holders and/or employees of Strata 534 Oncology.

Drs. Tomlins, Rhodes, Khazanov, and Johnson are named as co-inventors on a pending patent to Strata

537 Oncology related to the IRS model described herein.

538 Dr. Tomlins and Rhodes are equity holders in Javelin Oncology

539 Dr. Tomlins previously served as a consultant to Strata Oncology and has consulted for

540 Astellas/Medivation and Janssen. He has received research (to University of Michigan) funding from

541 Astellas and has received travel support from the Prostate Cancer Foundation.

542 Dr. Burkard reports receiving research funding from Abbvie, Genentech, Puma Biotechnology, Arcus

543 Biosciences, Apollomics, and Loxo Oncology.

544 Dr. Matrana reports receiving fees for serving on the speaker's bureau from Pfizer, Janssen, Astellas, 545 AstraZeneca Eisai Pharmaceuticals, Bristol-Myers Squibb, Genentech, SirTex, Merck and as a consultant 546 from AstraZeneca.

547 Dr. Yang reports receiving fees for serving on the advisory board from AstraZeneca, Bayer, Clovis 548 Oncology, and for receiving research support from Eli Lilly and Puma Biotechnology.

549 Dr. Parsons reports receiving fees for serving on the speaker's bureau and advisory board from Amgen 550 and Celgene, and for research funding from the Wisconsin Idea Grant, Gundersen Medical Foundation.

551 Dr. Thompson reports receiving consulting fees from Syapse Precision Medicine Council, Elsevier 552 ClinicalPath, Adaptive, UpToDate, GlaxoSmithKline, Takeda, Celgene, Doximity, and institutional 553 research funding from Abbvie, Bristol-Myers Squibb, CRAB CTC, Denovo, Hoosier Research Network, 554 Eli Lilly, LynxBio, Takeda, and TG Therapeutics.

555 Drs. Dees, Burkard, Matrana, and Yang received fees for serving on the Strata Oncology Clinical 556 Advisory Board.

557 Dr. Thompson reports receiving fees for Ad Boards with Sanofi

558 The remaining authors have no disclosures. 


\section{REFERENCES}

5611 Sharma, P. et al. The Next Decade of Immune Checkpoint Therapy. Cancer Discov 11, 838-857,

562

563

564

565

566

567

568

569

570

571

572

573

574

575

576

577

578

579

580

581

582

583

584

585

586

587

588

589

590

591

592

593

594

595

596

597

598

599

600

601

602

603

604

605

606

607

608

2 Chamoto, K., Hatae, R. \& Honjo, T. Current issues and perspectives in PD-1 blockade cancer immunotherapy. Int J Clin Oncol 25, 790-800, doi:10.1007/s10147-019-01588-7 (2020).

3 Ribas, A. \& Wolchok, J. D. Cancer immunotherapy using checkpoint blockade. Science 359, 1350-1355, doi:10.1126/science.aar4060 (2018).

4 Doroshow, D. B. et al. PD-L1 as a biomarker of response to immune-checkpoint inhibitors. Nat Rev Clin Oncol 18, 345-362, doi:10.1038/s41571-021-00473-5 (2021).

5 Gavrielatou, N., Shafi, S., Gaule, P. \& Rimm, D. L. PD-L1 Expression Scoring: NonInterchangeable, Non-Interpretable, Neither, or Both. J Natl Cancer Inst, doi:10.1093/jnci/djab109 (2021).

6 Rimm, D. L. et al. A Prospective, Multi-institutional, Pathologist-Based Assessment of 4 Immunohistochemistry Assays for PD-L1 Expression in Non-Small Cell Lung Cancer. JAMA Oncol 3, 1051-1058, doi:10.1001/jamaoncol.2017.0013 (2017).

7 Salgado, R. et al. How current assay approval policies are leading to unintended imprecision medicine. Lancet Oncol 21, 1399-1401, doi:10.1016/S1470-2045(20)30592-1 (2020).

8 Herbst, R. S. et al. Atezolizumab for First-Line Treatment of PD-L1-Selected Patients with NSCLC. N Engl J Med 383, 1328-1339, doi:10.1056/NEJMoa1917346 (2020).

9 Abdul Karim, L., Wang, P., Chahine, J. \& Kallakury, B. Harmonization of PD-L1 Immunohistochemistry Assays for Lung Cancer: A Working Progress. J Thorac Oncol 12, e45, doi:10.1016/j.jtho.2016.12.022 (2017).

10 Dolled-Filhart, M. et al. Development of a Prototype Immunohistochemistry Assay to Measure Programmed Death Ligand-1 Expression in Tumor Tissue. Arch Pathol Lab Med 140, 12591266, doi:10.5858/arpa.2015-0544-OA (2016).

11 Dolled-Filhart, M. et al. Development of a Companion Diagnostic for Pembrolizumab in NonSmall Cell Lung Cancer Using Immunohistochemistry for Programmed Death Ligand-1. Arch Pathol Lab Med 140, 1243-1249, doi:10.5858/arpa.2015-0542-OA (2016).

12 Hirsch, F. R. et al. PD-L1 Immunohistochemistry Assays for Lung Cancer: Results from Phase 1 of the Blueprint PD-L1 IHC Assay Comparison Project. J Thorac Oncol 12, 208-222, doi:10.1016/j.jtho.2016.11.2228 (2017).

13 Velcheti, V. et al. Real-world PD-L1 testing and distribution of PD-L1 tumor expression by immunohistochemistry assay type among patients with metastatic non-small cell lung cancer in the United States. PLoS One 13, e0206370, doi:10.1371/journal.pone.0206370 (2018).

14 Grant, M. J., Herbst, R. S. \& Goldberg, S. B. Selecting the optimal immunotherapy regimen in driver-negative metastatic NSCLC. Nat Rev Clin Oncol 18, 625-644, doi:10.1038/s41571-02100520-1 (2021).

15 Litchfield, K. et al. Meta-analysis of tumor- and T cell-intrinsic mechanisms of sensitization to checkpoint inhibition. Cell 184, 596-614 e514, doi:10.1016/j.cell.2021.01.002 (2021).

16 Rizvi, H. et al. Molecular Determinants of Response to Anti-Programmed Cell Death (PD)-1 and Anti-Programmed Death-Ligand 1 (PD-L1) Blockade in Patients With Non-Small-Cell Lung Cancer Profiled With Targeted Next-Generation Sequencing. J Clin Oncol 36, 633-641, doi:10.1200/JCO.2017.75.3384 (2018).

17 Singal, G. et al. Association of Patient Characteristics and Tumor Genomics With Clinical Outcomes Among Patients With Non-Small Cell Lung Cancer Using a Clinicogenomic Database. JAMA 321, 1391-1399, doi:10.1001/jama.2019.3241 (2019).

18 Rizvi, N. A. et al. Cancer immunology. Mutational landscape determines sensitivity to PD-1 blockade in non-small cell lung cancer. Science 348, 124-128, doi:10.1126/science.aaa1348 (2015). 
60919 McGranahan, N. et al. Clonal neoantigens elicit T cell immunoreactivity and sensitivity to

610

642

643

644

645

646

647

648

649

650

651

652

653

654

655

656

657

658 immune checkpoint blockade. Science 351, 1463-1469, doi:10.1126/science.aaf1490 (2016).

20 Van Allen, E. M. et al. Genomic correlates of response to CTLA-4 blockade in metastatic melanoma. Science 350, 207-211, doi:10.1126/science.aad0095 (2015).

21 Samstein, R. M. et al. Tumor mutational load predicts survival after immunotherapy across multiple cancer types. Nat Genet 51, 202-206, doi:10.1038/s41588-018-0312-8 (2019).

22 Vega, D. M. et al. Aligning tumor mutational burden (TMB) quantification across diagnostic platforms: phase II of the Friends of Cancer Research TMB Harmonization Project. Ann Oncol, doi:10.1016/j.annonc.2021.09.016 (2021).

23 Steuer, C. E. \& Ramalingam, S. S. Tumor Mutation Burden: Leading Immunotherapy to the Era of Precision Medicine? J Clin Oncol 36, 631-632, doi:10.1200/JCO.2017.76.8770 (2018).

24 Anagnostou, V. et al. Multimodal genomic features predict outcome of immune checkpoint blockade in non-small-cell lung cancer. Nat Cancer 1, 99-111, doi:10.1038/s43018-019-0008-8 (2020).

25 Marcus, L. et al. FDA Approval Summary: Pembrolizumab for the Treatment of Tumor Mutational Burden-High Solid Tumors. Clin Cancer Res 27, 4685-4689, doi:10.1158/10780432.CCR-21-0327 (2021).

26 Marabelle, A. et al. Association of tumour mutational burden with outcomes in patients with advanced solid tumours treated with pembrolizumab: prospective biomarker analysis of the multicohort, open-label, phase 2 KEYNOTE-158 study. Lancet Oncol 21, 1353-1365, doi:10.1016/S1470-2045(20)30445-9 (2020).

27 Cristescu, R. et al. Pan-tumor genomic biomarkers for PD-1 checkpoint blockade-based immunotherapy. Science 362, doi:10.1126/science.aar3593 (2018).

28 Lee, J. S. \& Ruppin, E. Multiomics Prediction of Response Rates to Therapies to Inhibit Programmed Cell Death 1 and Programmed Cell Death 1 Ligand 1. JAMA Oncol 5, 1614-1618, doi:10.1001/jamaoncol.2019.2311 (2019).

29 Rolfo, C. et al. Liquid Biopsy for Advanced NSCLC: A Consensus Statement From the International Association for the Study of Lung Cancer. J Thorac Oncol 16, 1647-1662, doi:10.1016/j.jtho.2021.06.017 (2021).

30 Herbst, R. S. et al. Predictive correlates of response to the anti-PD-L1 antibody MPDL3280A in cancer patients. Nature 515, 563-567, doi:10.1038/nature14011 (2014).

31 Taube, J. M. et al. Colocalization of inflammatory response with B7-h1 expression in human melanocytic lesions supports an adaptive resistance mechanism of immune escape. Sci Transl Med 4, 127ra137, doi:10.1126/scitranslmed.3003689 (2012).

32 Chen, D. S. \& Mellman, I. Elements of cancer immunity and the cancer-immune set point. Nature 541, 321-330, doi:10.1038/nature21349 (2017).

33 Sanmamed, M. F. \& Chen, L. A Paradigm Shift in Cancer Immunotherapy: From Enhancement to Normalization. Cell 175, 313-326, doi:10.1016/j.cell.2018.09.035 (2018).

34 Ayers, M. et al. IFN-gamma-related mRNA profile predicts clinical response to PD-1 blockade. $J$ Clin Invest 127, 2930-2940, doi:10.1172/JCI91190 (2017).

35 Fountzilas, E., Kurzrock, R., Hiep Vo, H. \& Tsimberidou, A. M. Wedding of Molecular Alterations and Immune Checkpoint Blockade: Genomics as a Matchmaker. J Natl Cancer Inst, doi:10.1093/jnci/djab067 (2021).

36 Ott, P. A. et al. T-Cell-Inflamed Gene-Expression Profile, Programmed Death Ligand 1 Expression, and Tumor Mutational Burden Predict Efficacy in Patients Treated With Pembrolizumab Across 20 Cancers: KEYNOTE-028. J Clin Oncol 37, 318-327, doi:10.1200/JCO.2018.78.2276 (2019).

37 Lu, S. et al. Comparison of Biomarker Modalities for Predicting Response to PD-1/PD-L1 Checkpoint Blockade: A Systematic Review and Meta-analysis. JAMA Oncol 5, 1195-1204, doi:10.1001/jamaoncol.2019.1549 (2019). 
661

662

663

664

665

666

667

668

669

670

671

672

673

674

675

676

677

678

679

680

681

682

683

684

685

686

687

688

689

690

691

692

693

694

695

696

697

698

699

700

701

702

703

704

705

706

707

708
Zhang, Z. et al. RNF2 ablation reprograms the tumor-immune microenvironment and stimulates durable NK and CD4+ T-cell-dependent antitumor immunity. Nature Cancer 2, 1018-1038, doi:10.1038/s43018-021-00263-z (2021).

39 Cristescu, R. et al. Transcriptomic Determinants of Response to Pembrolizumab Monotherapy Across Solid Tumor Types. Clin Cancer Res, doi:10.1158/1078-0432.CCR-21-3329 (2021).

40 Harms, K. L. et al. Virus-positive Merkel Cell Carcinoma Is an Independent Prognostic Group with Distinct Predictive Biomarkers. Clin Cancer Res 27, 2494-2504, doi:10.1158/10780432.CCR-20-0864 (2021).

41 Tomlins, S. A. et al. Development and Validation of StrataNGS, a Multiplex PCR, Semiconductor Sequencing-Based Comprehensive Genomic Profiling Test. J Mol Diagn 23, 1515-1533, doi:10.1016/j.jmoldx.2021.08.005 (2021).

42 Tomlins, S. A. et al. Real-World Performance of a Comprehensive Genomic Profiling Test Optimized for Small Tumor Samples. JCO Precis Oncol 5, doi:10.1200/PO.20.00472 (2021).

43 Zehir, A. et al. Mutational landscape of metastatic cancer revealed from prospective clinical sequencing of 10,000 patients. Nat Med 23, 703-713, doi:10.1038/nm.4333 (2017).

44 van de Haar, J. et al. Limited evolution of the actionable metastatic cancer genome under therapeutic pressure. Nat Med 27, 1553-1563, doi:10.1038/s41591-021-01448-w (2021).

45 Medicine, F. (2021).

46 Yearley, J. H. et al. PD-L2 Expression in Human Tumors: Relevance to Anti-PD-1 Therapy in Cancer. Clin Cancer Res 23, 3158-3167, doi:10.1158/1078-0432.CCR-16-1761 (2017).

47 Giraldo, N. A. et al. Multidimensional, quantitative assessment of PD-1/PD-L1 expression in patients with Merkel cell carcinoma and association with response to pembrolizumab. $J$

Immunother Cancer 6, 99, doi:10.1186/s40425-018-0404-0 (2018).

48 Garon, E. B. et al. Pembrolizumab for the treatment of non-small-cell lung cancer. $N$ Engl J Med 372, 2018-2028, doi:10.1056/NEJMoa1501824 (2015).

49 Robert, C. et al. Pembrolizumab versus Ipilimumab in Advanced Melanoma. N Engl J Med 372, 2521-2532, doi:10.1056/NEJMoa1503093 (2015).

50 Tumeh, P. C. et al. PD-1 blockade induces responses by inhibiting adaptive immune resistance. Nature 515, 568-571, doi:10.1038/nature13954 (2014).

51 Mahoney, K. M. \& Atkins, M. B. Prognostic and predictive markers for the new immunotherapies. Oncology (Williston Park) 28 Suppl 3, 39-48 (2014).

$52 \mathrm{Yu}, \mathrm{C}$. et al. A five-gene signature is a prognostic biomarker in pan-cancer and related with immunologically associated extracellular matrix. Cancer Med 10, 4629-4643, doi:10.1002/cam4.3986 (2021).

53 Liu, Y. et al. ADAM12 is a costimulatory molecule that determines Th1 cell fate and mediates tissue inflammation. Cell Mol Immunol 18, 1904-1919, doi:10.1038/s41423-020-0486-8 (2021).

54 Cheon, D. J. et al. ADAM12 is a prognostic factor associated with an aggressive molecular subtype of high-grade serous ovarian carcinoma. Carcinogenesis 36, 739-747, doi:10.1093/carcin/bgv059 (2015).

55 Cui, C. et al. Ratio of the interferon-gamma signature to the immunosuppression signature predicts anti-PD-1 therapy response in melanoma. NPJ Genom Med 6, 7, doi:10.1038/s41525021-00169-w (2021).

56 Ruff, M. et al. The Disintegrin and Metalloprotease ADAM12 Is Associated with TGF-betaInduced Epithelial to Mesenchymal Transition. PLoS One 10, e0139179, doi:10.1371/journal.pone.0139179 (2015).

57 Atfi, A. et al. The disintegrin and metalloproteinase ADAM12 contributes to TGF-beta signaling through interaction with the type II receptor. J Cell Biol 178, 201-208, doi:10.1083/jcb.200612046 (2007).

58 Pelka, K. et al. Spatially organized multicellular immune hubs in human colorectal cancer. Cell 184, 4734-4752 e4720, doi:10.1016/j.cell.2021.08.003 (2021). 
59 Mariathasan, S. et al. TGFbeta attenuates tumour response to PD-L1 blockade by contributing to exclusion of T cells. Nature 554, 544-548, doi:10.1038/nature25501 (2018).

60 Tauriello, D. V. F. et al. TGFbeta drives immune evasion in genetically reconstituted colon cancer metastasis. Nature 554, 538-543, doi:10.1038/nature25492 (2018).

61 Jiang, P. et al. Signatures of T cell dysfunction and exclusion predict cancer immunotherapy response. Nat Med 24, 1550-1558, doi:10.1038/s41591-018-0136-1 (2018).

62 Bonaventura, P. et al. Cold Tumors: A Therapeutic Challenge for Immunotherapy. Front Immunol 10, 168, doi:10.3389/fimmu.2019.00168 (2019).

63 Gajewski, T. F., Schreiber, H. \& Fu, Y. X. Innate and adaptive immune cells in the tumor microenvironment. Nat Immunol 14, 1014-1022, doi:10.1038/ni.2703 (2013).

64 Cottrell, T. R. \& Taube, J. M. PD-L1 and Emerging Biomarkers in Immune Checkpoint Blockade Therapy. Cancer J 24, 41-46, doi:10.1097/PPO.0000000000000301 (2018).

65 Kerr, K. M. The PD-L1 Immunohistochemistry Biomarker: Two Steps Forward, One Step Back? J Thorac Oncol 13, 291-294, doi:10.1016/j.jtho.2018.01.020 (2018).

66 Sholl, L. M. et al. The Promises and Challenges of Tumor Mutation Burden as an Immunotherapy Biomarker: A Perspective from the International Association for the Study of Lung Cancer Pathology Committee. J Thorac Oncol 15, 1409-1424, doi:10.1016/j.jtho.2020.05.019 (2020).

67 Addeo, A., Friedlaender, A., Banna, G. L. \& Weiss, G. J. TMB or not TMB as a biomarker: That is the question. Crit Rev Oncol Hematol 163, 103374, doi:10.1016/j.critrevonc.2021.103374 (2021).

68 Han, G. et al. 9p21 loss confers a cold tumor immune microenvironment and primary resistance to immune checkpoint therapy. Nat Commun 12, 5606, doi:10.1038/s41467-021-25894-9 (2021).

69 Sholl, L. M. Biomarkers of response to checkpoint inhibitors beyond PD-L1 in lung cancer. Mod Pathol, doi:10.1038/s41379-021-00932-5 (2021).

70 Di Federico, A., De Giglio, A., Parisi, C. \& Gelsomino, F. STK11/LKB1 and KEAP1 mutations in non-small cell lung cancer: Prognostic rather than predictive? Eur J Cancer 157, 108-113, doi:10.1016/j.ejca.2021.08.011 (2021).

71 Skoulidis, F. et al. STK11/LKB1 Mutations and PD-1 Inhibitor Resistance in KRAS-Mutant Lung Adenocarcinoma. Cancer Discov 8, 822-835, doi:10.1158/2159-8290.CD-18-0099 (2018).

72 Shen, J. et al. ARID1A deficiency promotes mutability and potentiates therapeutic antitumor immunity unleashed by immune checkpoint blockade. Nat Med 24, 556-562, doi:10.1038/s41591018-0012-z (2018).

73 Miao, D. et al. Genomic correlates of response to immune checkpoint therapies in clear cell renal cell carcinoma. Science 359, 801-806, doi:10.1126/science.aan5951 (2018).

74 Braun, D. A. et al. Clinical Validation of PBRM1 Alterations as a Marker of Immune Checkpoint Inhibitor Response in Renal Cell Carcinoma. JAMA Oncol 5, 1631-1633, doi:10.1001/jamaoncol.2019.3158 (2019).

75 Liu, X. D. et al. PBRM1 loss defines a nonimmunogenic tumor phenotype associated with checkpoint inhibitor resistance in renal carcinoma. Nat Commun 11, 2135, doi:10.1038/s41467020-15959-6 (2020).

76 Banchereau, R. et al. Molecular determinants of response to PD-L1 blockade across tumor types. Nat Commun 12, 3969, doi:10.1038/s41467-021-24112-w (2021). 


\section{FIGURE LEGENDS}

\section{Figure 1. An integrative Immune Response Score (IRS) model predicts real-world pembrolizumab} treatment outcome across solid tumors. a) The tumor type distribution of 708 patients in the StrataTrial (NCT03061305) clinical molecular database used to develop the IRS model, an integrative algorithm predicting pembrolizumab benefit. Included patients were treated with pembrolizumab and had available tumor mutation burden (from comprehensive genomic profiling) and in-parallel quantitative gene expression data of immune relevant biomarkers from clinical testing of routine tumor tissue. b) Realworld pembrolizumab progression-free survival, stratified by IRS group. After IRS model development, patients were binned into three groups based on predicted pembrolizumab benefit: IRS-Low (grey line), IRS-Intermediate (inter., light blue line), and IRS-High (dark blue line). Real world progression free survival was determined using time to next therapy (TTNT; see Methods). c) Confirmation of the predictive nature of the IRS model. Real-world progression-free survival on monotherapy pembrolizumab (Pembro; purple) vs. immediately preceding chemotherapy (Chemo; line green) stratified by IRS group was compared for the applicable subset of 166 patients. The interaction test for continuous IRS and pembrolizumab vs. chemotherapy treatment was significant (likelihood ratio test for interaction $\mathrm{p}<0.005$ ). 


\section{Figure 1}

a

b

Immune Response Score Cohort

$(\mathrm{N}=708)$

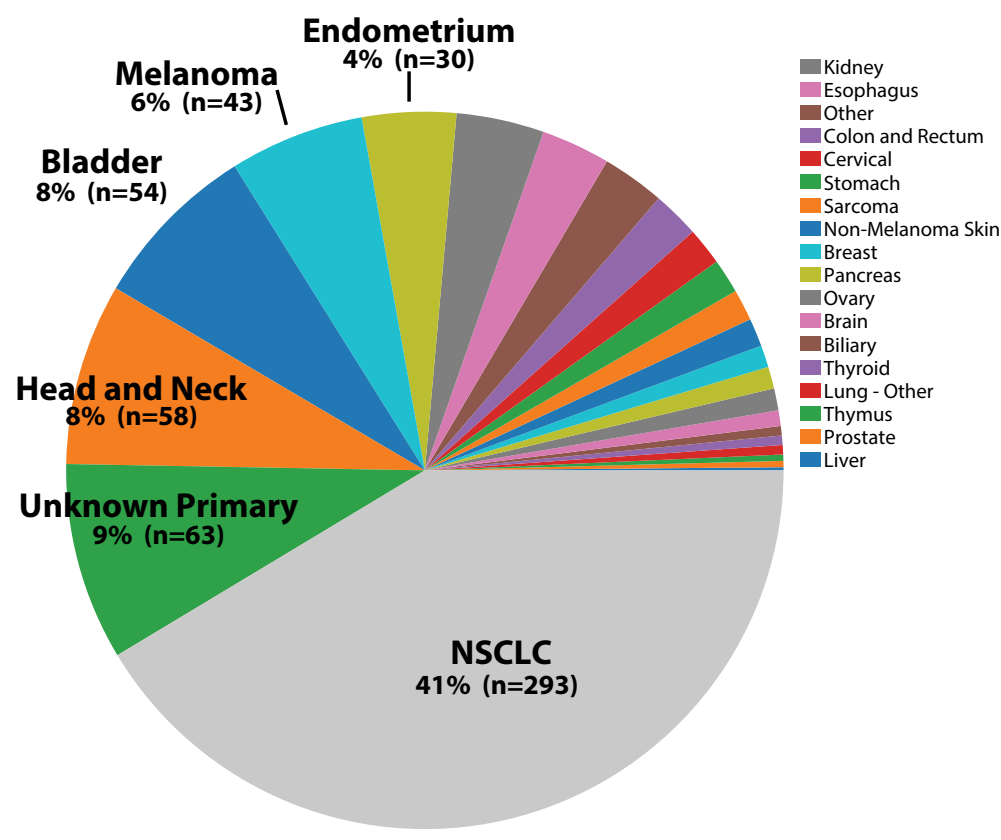

Pembro TTNT

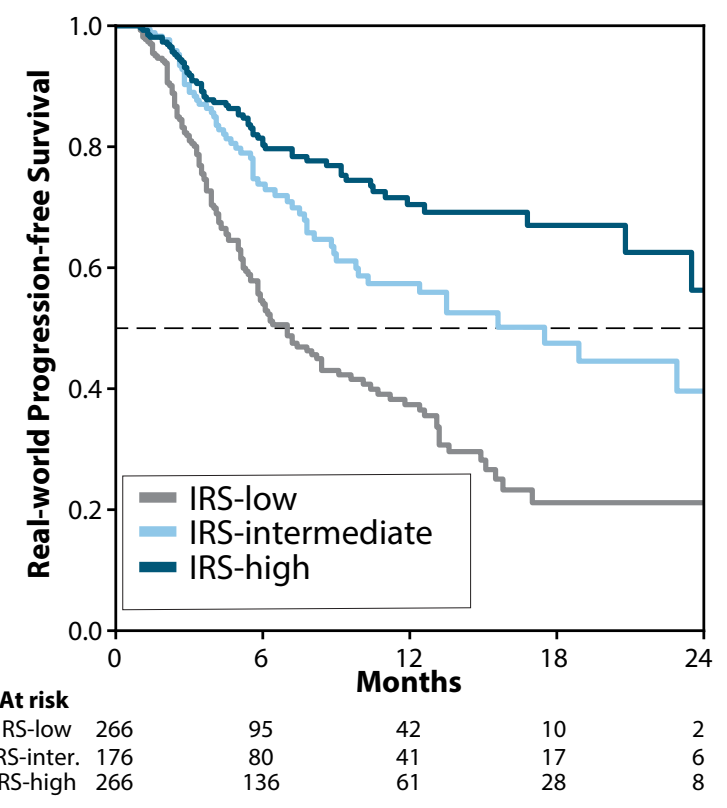

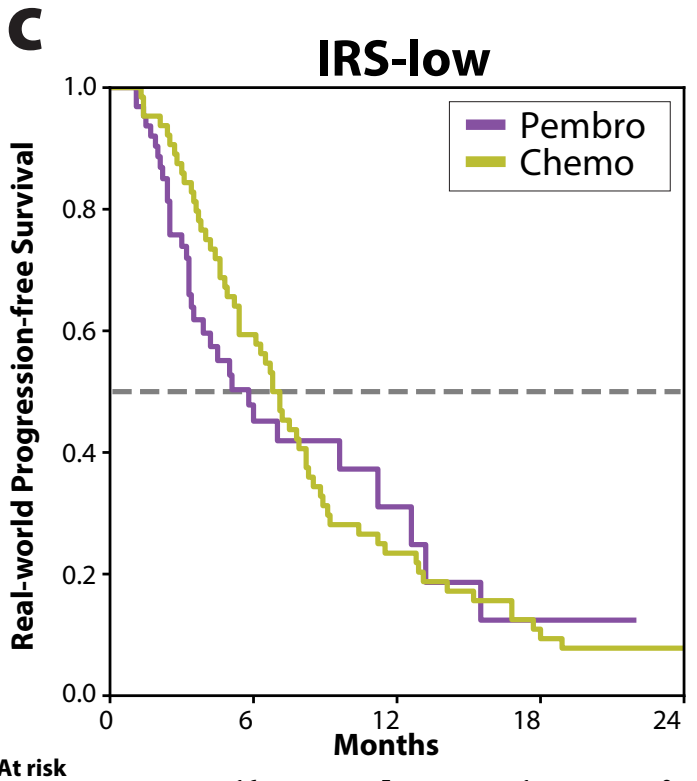
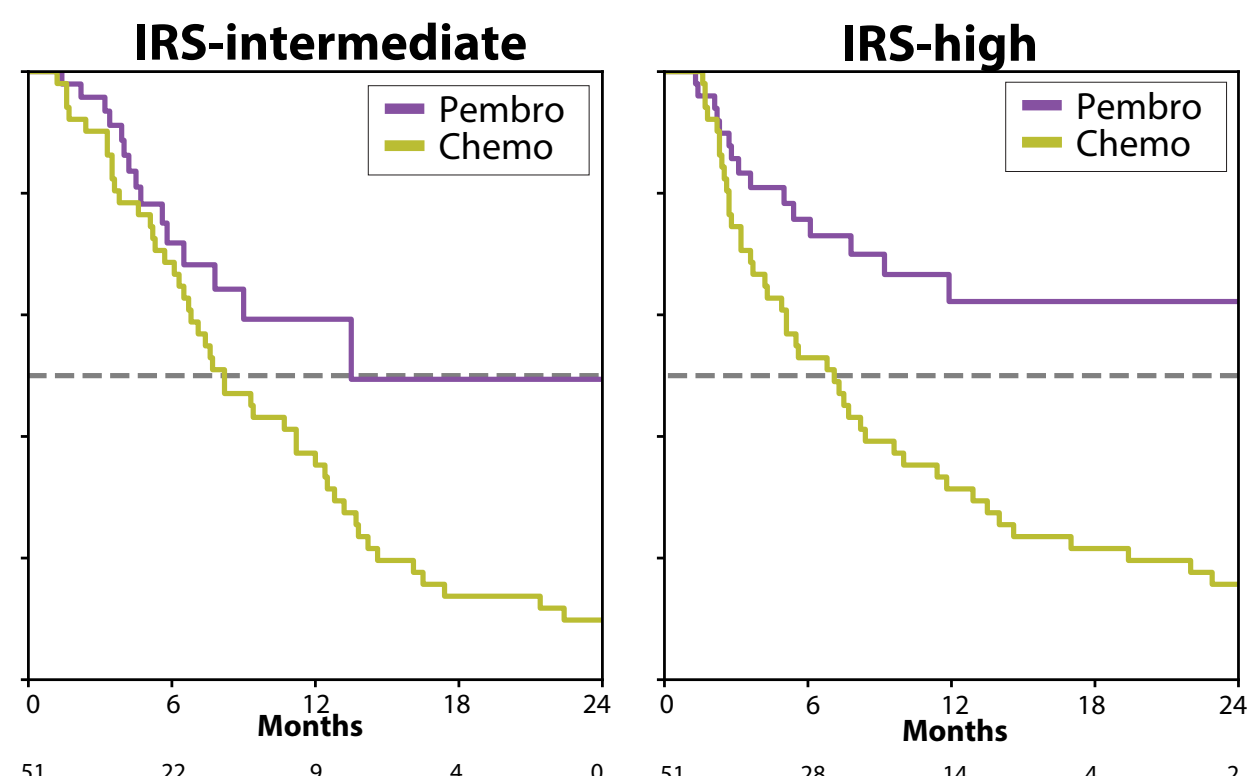

Pembro $\begin{array}{ll}64 & 16 \\ 64 & 38\end{array}$

5
15

$\begin{array}{lll}1 & 0 & 51 \\ 6 & 5 & 51\end{array}$

9
18

7

28
27

14
16 
769 Figure 2. Robustness of the IRS model to potential confounding factors. a) Real-world progression-

770 free survival on pembrolizumab in patients with non-small cell lung cancer (NSCLC, blue) versus other

771 tumor types (Other, lime green), stratified by IRS group. b) Real-world progression-free survival on

772 pembrolizumab in patients treated with pembrolizumab monotherapy (mono, blue) versus pembrolizumab

773 + chemotherapy combination (combo, lime green), stratified by IRS group. c) Real-world progression-

774 free survival on pembrolizumab in TMB-low and TMB-high patients, stratified by IRS groups (IRS-low

775 [grey], IRS-intermediate [light blue], or IRS-high [dark blue line]). TMB, tumor mutation burden. 


\section{Figure 2}

a
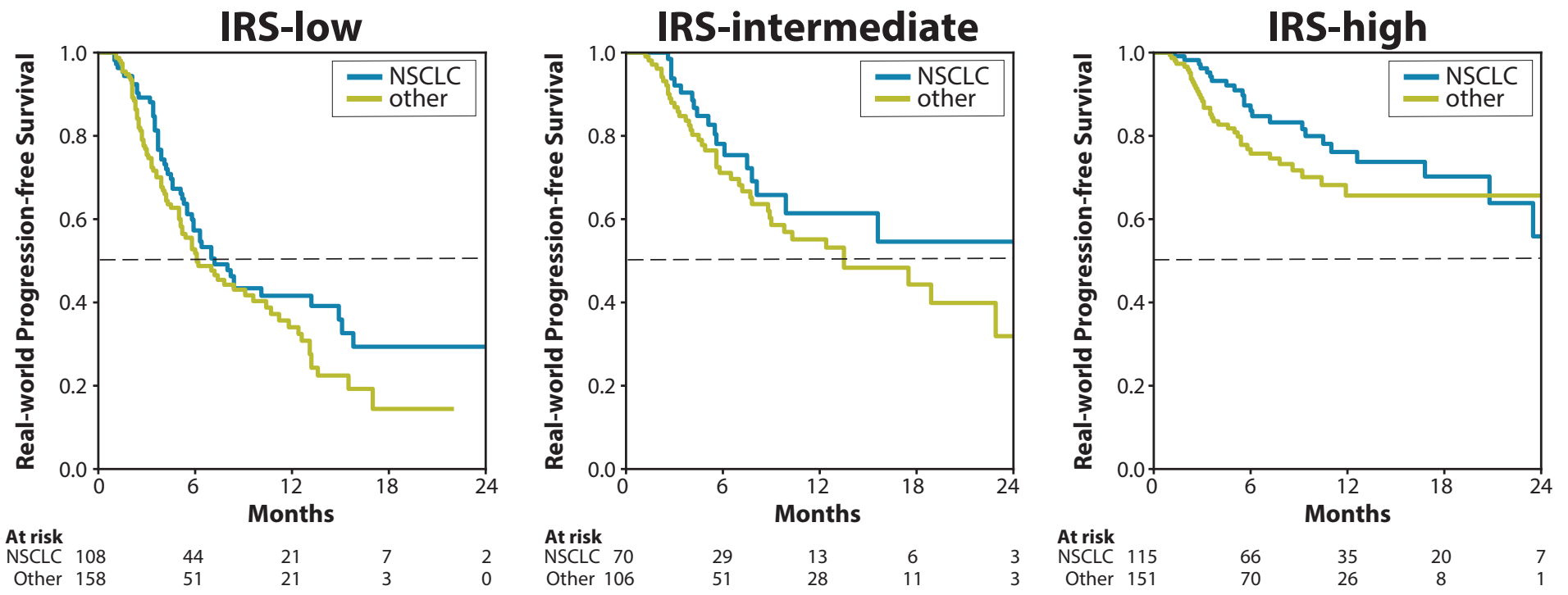

b

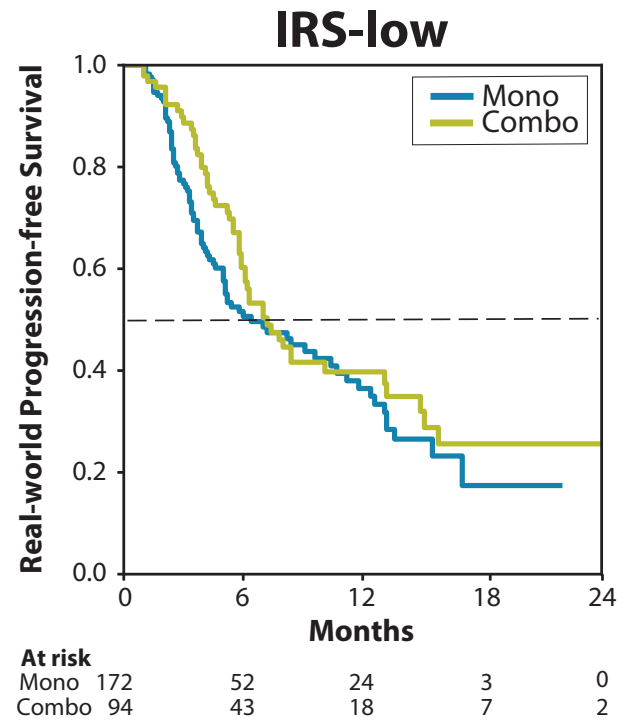

C

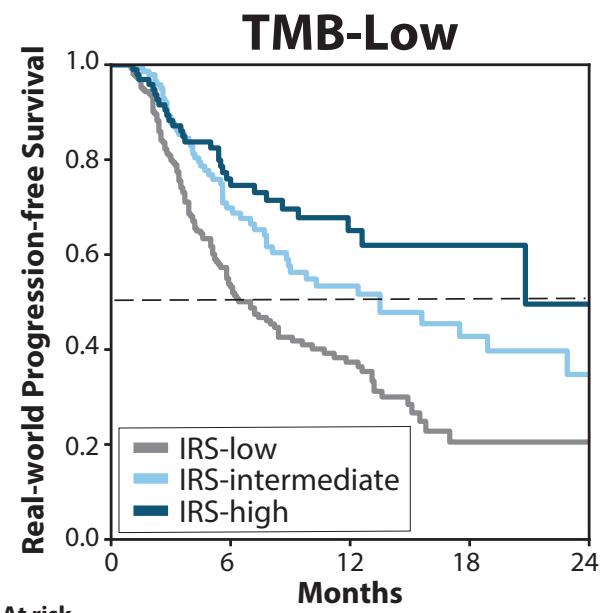

IRS-low 253

IRS-intermediate 148

IRS-high 99

$\begin{array}{llcl}87 & 39 & 9 & 2 \\ 65 & 32 & 15 & 5 \\ 54 & 24 & 10 & 1\end{array}$
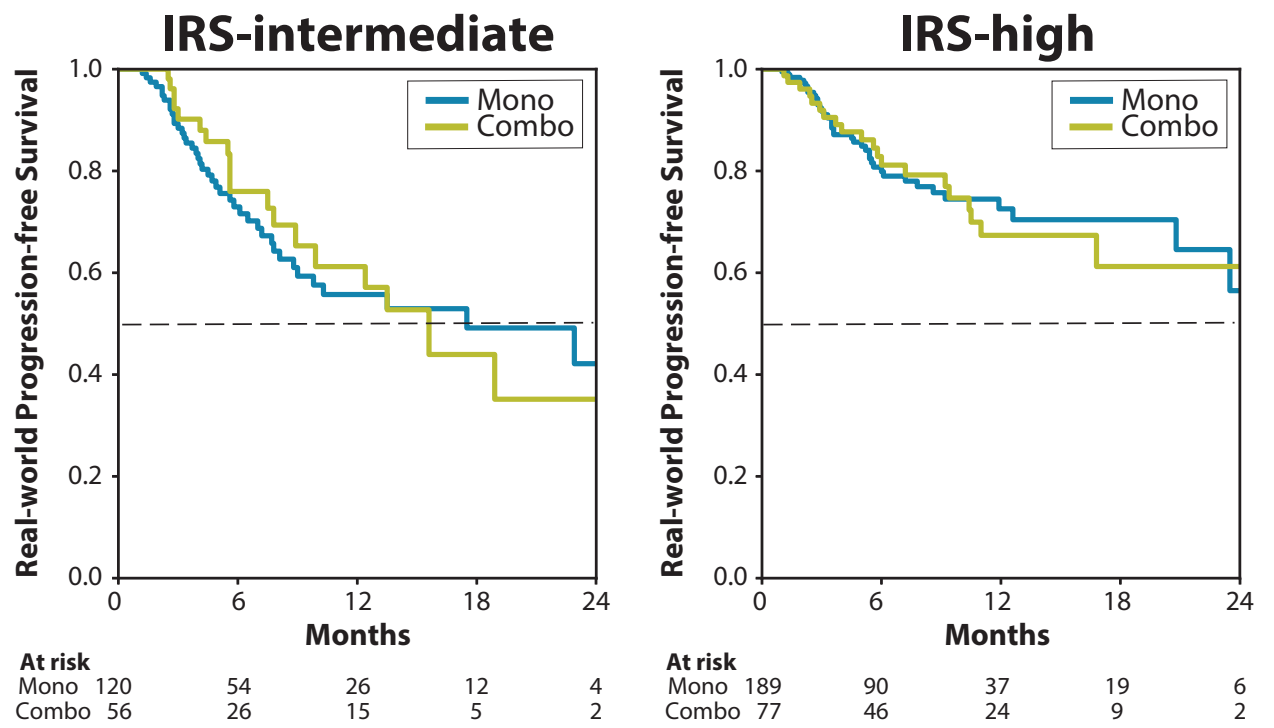

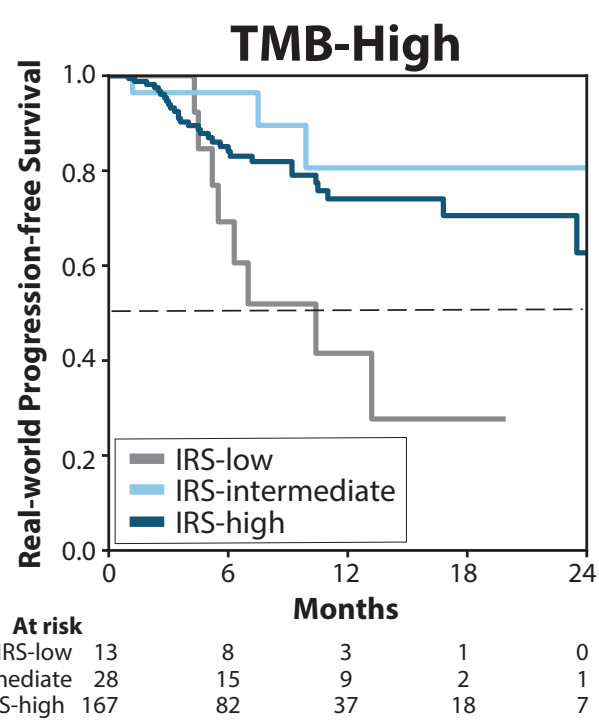



assess potential clinical utility. a) IRS groups were determined for all 25,770 patients in the SCMD with

779 valid TMB and gene expression data. The distribution by IRS group (IRS-low [grey], IRS-intermediate

780 [light blue], and IRS-high [dark blue]) is shown. b) IRS distribution by pembrolizumab approved vs. not 781 not-approved tumor types. c) Stratification of b) by tumor type. d) IRS distribution by TMB-high vs.

782 TMB-low. e) IRS distribution by any pembrolizumab approved indication (approved tumor type, MSI-H 783 or TMB-H as approved) vs. not approved indication. Pembro, Pembrolizumab, TMB, Tumor Mutation

784 Burden; CRC, colorectal cancer; CUP, cancer of unknown primary; Lung- Other, lung small cell

785 carcinoma; NSCLC, non-small cell lung cancer; NMSC, nonmelanoma skin cancer; Small Intes., small 786 intestine. Numbers in subpanels may not add to the totals in a) due to rounding. 
a

25,770 subjects in SCMD

\begin{tabular}{l|l|l|l|} 
& & & \\
Total & 13.2 & 17.5 & 69.3 \\
\hline
\end{tabular}

b Pembro Not-Approved Pembro Approved
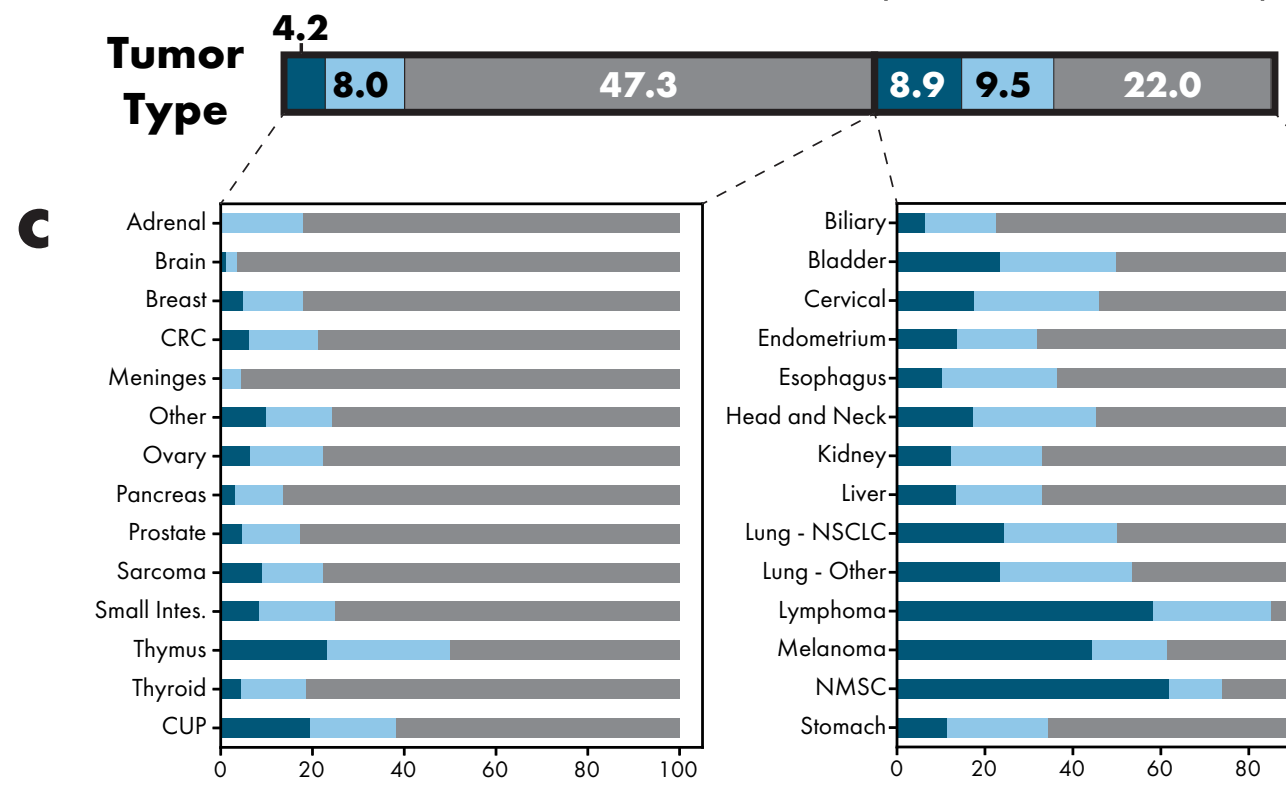

d
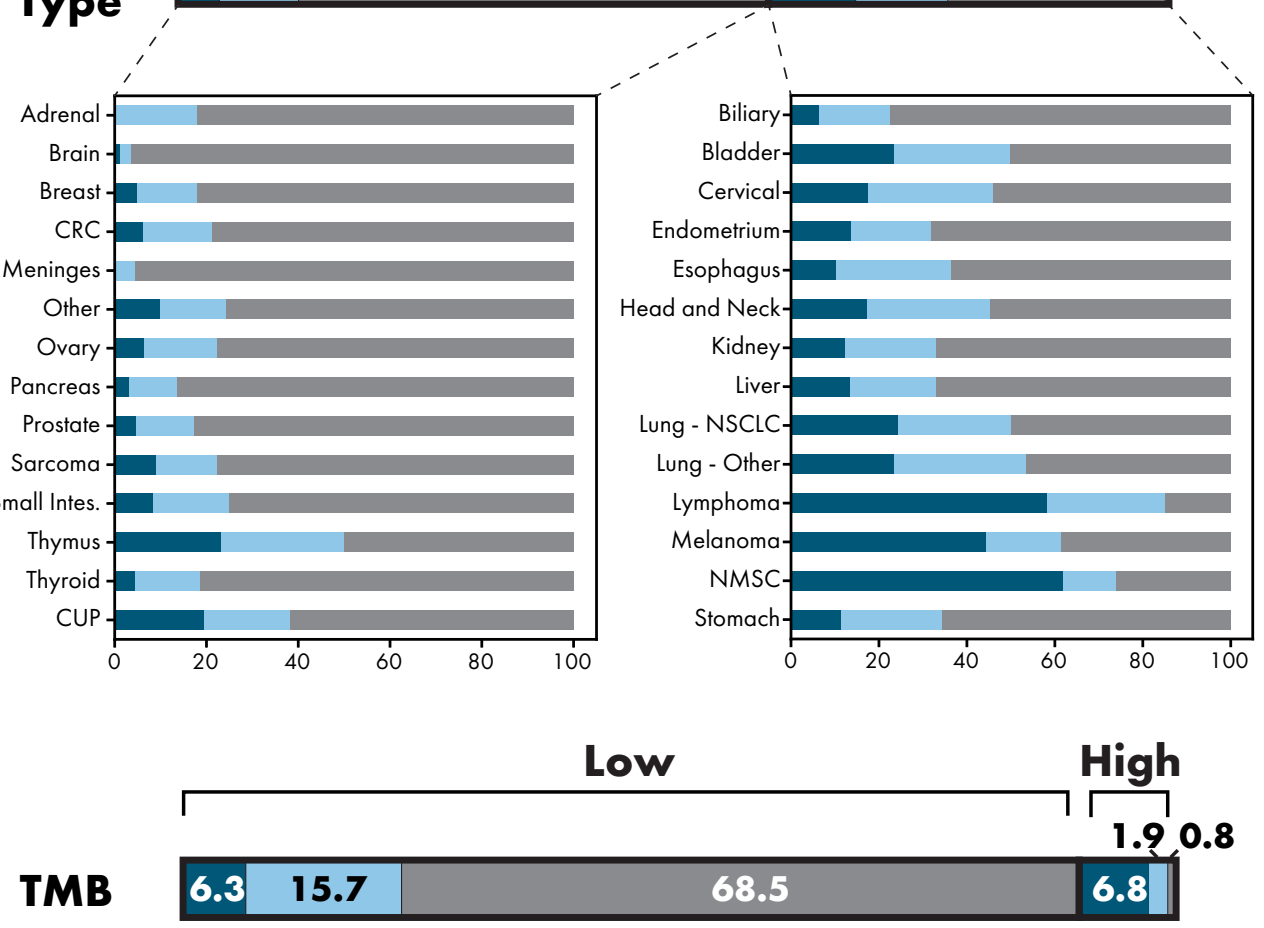

e

\begin{tabular}{|c|c|c|c|c|c|}
\hline & \multicolumn{2}{|c|}{ Not Approved } & \multicolumn{3}{|c|}{ Approved } \\
\hline All & 2.2 & & & & \\
\hline Pembro & 7.4 & 47.0 & 11.0 & 10.0 & 22.3 \\
\hline
\end{tabular}
Indications

\begin{tabular}{|l|l|}
\hline IRS-high IRS-intermediate I I & IRS
\end{tabular}




\section{TABLES}

Table 1. Univariate and multivariate associations of comprehensive genomic and quantitative transcriptomic profiling derived candidate biomarkers and real world pembrolizumab progression free survival in 708 patients

\begin{tabular}{|c|c|c|c|c|}
\hline \multirow[t]{2}{*}{ Biomarker } & \multicolumn{2}{|c|}{ Univariate } & \multicolumn{2}{|c|}{ Multivariate (IRS model) } \\
\hline & HR $(95 \%$ CI $)$ & $\mathbf{p}$ & HR & $\mathbf{p}$ \\
\hline TMB & $0.77(0.70-28.02)$ & $3.7 \mathrm{E}-09$ & $0.75(0.69-0.82)$ & $5.8 \mathrm{E}-10$ \\
\hline$C D 274(P D-L 1)$ Composite & $0.90(0.85-10.47)$ & $7.1 \mathrm{E}-04$ & & \\
\hline PDCD1 (PD-1) Composite & $0.89(0.84-9.83)$ & $1.1 \mathrm{E}-03$ & $0.86(0.79-0.94)$ & $5.0 \mathrm{E}-04$ \\
\hline$P D C D 1 L G 2(P D-L 2)$ & $0.91(0.85-7.77)$ & $4.6 \mathrm{E}-03$ & $0.87(0.79-0.95)$ & $1.3 \mathrm{E}-03$ \\
\hline TNFRSF9 & $0.92(0.87-7.07)$ & $7.4 \mathrm{E}-03$ & & \\
\hline$I D O I$ & $0.95(0.92-5.42)$ & 0.023 & & \\
\hline$U B E 2 C^{*}$ & $0.91(0.83-5.09)$ & 0.029 & & \\
\hline TIGIT & $0.95(0.90-3.51)$ & 0.088 & & \\
\hline$L A G 3$ & $0.94(0.88-3.19)$ & 0.109 & & \\
\hline$C D 8 A$ & $0.95(0.90-3.17)$ & 0.111 & & \\
\hline$I F N G$ & $0.98(0.95-2.51)$ & 0.176 & & \\
\hline TCF7 & $0.95(0.87-2.35)$ & 0.196 & & \\
\hline CTLA4 & $0.97(0.92-2.10)$ & 0.233 & & \\
\hline GZMA & $0.97(0.91-1.57)$ & 0.337 & & \\
\hline TOP $2 A^{*}$ & $0.96(0.87-1.49)$ & 0.356 & & \\
\hline VTCN1 & $0.99(0.96-1.28)$ & 0.413 & & \\
\hline FOXP3 & $0.98(0.92-1.19)$ & 0.439 & & \\
\hline ADAM12 & $1.02(0.95-0.69)$ & 0.620 & $1.08(1.01-1.15)$ & 0.03 \\
\hline$C D 4$ & $1.02(0.93-0.63)$ & 0.646 & $1.15(1.01-1.31)$ & 0.04 \\
\hline$A X L$ & $0.98(0.90-0.52)$ & 0.699 & & \\
\hline HAVCR2 & $1.00(0.93-0.11)$ & 0.929 & & \\
\hline TNFRSF4 & $1.00(0.93-0.08)$ & 0.946 & & \\
\hline
\end{tabular}

For each biomarker, the hazard ratio (with $95 \%$ confidence interval bounds) and log-likelihood p-value are shown. TMB $\left(\log _{2}\right)$ was from StrataNGS CGP testing; the remaining biomarkers were target gene expression from in-parallel quantitative transcriptomic profiling by Multiplex PCR-based RNA sequencing. The multivariate analysis was performed using the final five component Immune Response Score (IRS) model. Candidate proliferation markers are indicated by *. For $P D-L 1$ and $P D-1$, two independent target amplicons were assessed for each gene; normalized target gene expression was averaged from the independent amplicons (per gene) to yield a composite result. 


\section{Supplementary Files}

This is a list of supplementary files associated with this preprint. Click to download.

- IRSSuppfinal.pdf 